U.S. DEPARTMENT OF THE INTERIOR

U.S. GEOLOGICAL SURVEY

Cape Hatteras to the Mid-Atlantic Ridge --

Demultiplexing and Archiving

A Unique Multichannel Seismic Reflection Data Set

by

W.F. Agena ${ }^{1}$, D.R. Hutchinson ${ }^{2}$, M.W. Lee ${ }^{1}$, and H.L. Oliver ${ }^{1}$

U.S. Geological Survey

Open-File Report 93-264

This report is preliminary and has not been reviewed for conformity with U.S. Geological Survey editorial standards and stratigraphic nomenclature. Any use of trade names is for descriptive purposes only and does not imply endorsement by the U.S. Geological Survey.

${ }^{1}$ U.S. Geological Survey, Box 25046, MS 960, DFC, Denver, CO 80225

${ }^{2}$ U.S. Geological Survey, Woods Hole, MA 02543 
Table of Contents

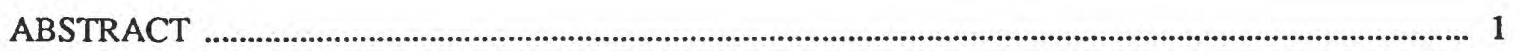

INTRODUCTION …................................................................................................................................. 1

DEMULTIPLEXING AND COMPRESSION .......................................................................................... 1

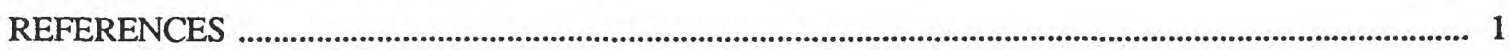

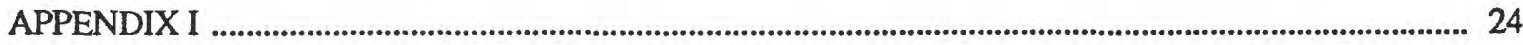

Figures

Figure 1 - Shotpoint map of survey area ................................................................................................. 2

Figure 2 - Shotpoint map of survey area near Cape Hatteras ........................................................................... 3

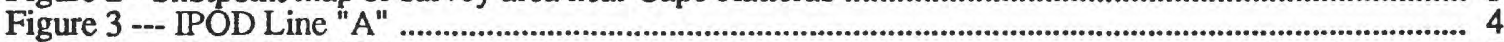

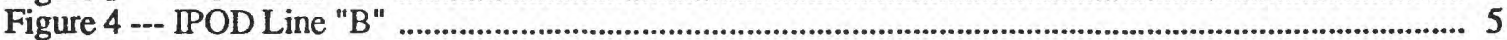

Figure 5 --- IPOD Line "1" Shotpoints 101-1000 ….............................................................................. 6

Figure 6 --- IPOD Line "1" Shotpoints 1000-2000 …......................................................................... 7

Figure 7 --- IPOD Line "1" Shotpoints 2000-4000 _....................................................................................... 8

Figure 8 --- IPOD Line "1" Shotpoints 4000-6000 ................................................................................. 9

Figure 9 --- IPOD Line "1" Shotpoints 6000-8000 .......................................................................................... 10

Figure 10 --- IPOD Line "1" Shotpoints 8000-10000 ........................................................................ 11

Figure 11 --- IPOD Line "1" Shotpoints 10000-12000 .......................................................................... 12

Figure 12 --- IPOD Line " " "Shotpoints 12000-14000 ................................................................................... 13

Figure 13 --- IPOD Line "1" Shotpoints 14000-16000 .................................................................................. 14

Figure 14 -.- IPOD Line "1" Shotpoints 16000-18000 ........................................................................................... 15

Figure 15 --- IPOD Line "1" Shotpoints 18000-20000 ................................................................................. 16

Figure 16 --- IPOD Line "1" Shotpoints 20000-22000 ....................................................................................... 17

Figure 17 --- IPOD Line "1" Shotpoints 22000-24000 ........................................................................... 18

Figure 18 --- IPOD Line "1" Shotpoints 24000-26000 ................................................................................ 19

Figure 19 --- IPOD Line "1" Shotpoints 26000-28000 …….............................................................................. 20

Figure 20 --- IPOD Line "1" Shotpoints 28000-30000 .......................................................................... 21

Figure 21 --- IPOD Line "1" Shotpoints 30000-32000 .................................................................... 22

Figure 22 --- IPOD Line "1" Shotpoints 32000-34110 ........................................................................... 23

Tables

Table 1 - Field recording parameters ....................................................................................................... 3 


\title{
Cape Hatteras to the Mid-Atlantic Ridge -- Demultiplexing and Archiving A Unique Multichannel Seismic Reflection Data Set
}

\author{
by \\ W.F. Agena, D.R. Hutchinson, M.W. Lee, and H.L. Oliver
}

\begin{abstract}
We have demultiplexed and compressed data from a 3400-km-long multichannel seismic-reflection line collected between Cape Hatteras to the Mid-Allantic Ridge in a continuing effort to reduce the amount of data loss due to old, deteriorating magnetic tapes.

\section{INTRODUCTION}

In 1974, Digicon Inc. recorded a $3400 \mathrm{~km}$-long multichannel seismic-reflection profile (Line 1) extending from Cape Hatteras to the rift valley of the Mid-Atlantic Ridge (Figure 1). Also acquired simultaneously were two small lines totalling $80 \mathrm{~km}$ (Lines A and B), connecting the main line to two coastal wells in North Carolina (Figure 2.) Jointly funded by the U.S. Geological Survey (USGS) and the National Science Foundation's Deep Sea Drilling Project (DSDP), the experiment was part of the International Phase of Ocean Drilling (IPOD). The data were recorded from the marine vessel Gulf Seal using a Texas Instruments DFS III recording system. A tuned airgun array totaling 1,700 in. $^{3}$ was used as the seismic source and a streamer with 48-non-linearly spaced hydrophone groups was used to detect the returning seismic signals. Shotpoint locations, generally spaced every 50 meters, are shown in Figures 1 and 2. A summary of the shooting and recording parameters is shown in Table 1. Segments of the western half of the data totalling approximately $1,520 \mathrm{~km}$, or just under half of the line, were processed by Geophysical Services, Inc. (GSI) in the spring of 1975. A geologic overview of this line can be found in Grow and Markl (1977). Copies of the demultiplexed field tapes are currently stored at the USGS seismic data processing center in Denver, Colorado.
\end{abstract}

\section{DEMULTIPLEXING AND COMPRESSION}

Testing revealed that the data on our original field tapes were deteriorating. In order to prevent further loss of data, we demultiplexed the original 631 field tapes and transfered the output to 183 new magnetic tapes at 6250 bpi in SEG-Y (32-bit IBM floating-point) format, an industry accepted standard (Barry and others, 1975) Our efforts yielded a data compression ratio of more than 3 to 1 . Although there were segments on some of the field tapes where the magnetic oxide had actually fallen off the tapes, we preserved as much of the data as possible. Close monitoring of the processing system during demultiplexing enabled the computer operators to prevent any further data loss (or misreading of data) due to parity read errors. When exessive parity errors were found on input tapes, operators either passed the tapes through a tape cleaner or, in more severe cases, made a bit-for-bit tape copy. Less than .2 percent of the original data could not be recovered during demultiplexing. Shown in appendix I are the slot numbers, shotpoint and field file ranges of all the output tapes. Slot numbers refer to storage locations numbers within the Denver tape library and reel numbers refer to the database reference number of our processing system. Observer's notes are also archived at the Denver processing center and can be obtained from the authors.

In order to help users better locate points of interest along the IPOD lines, we have included displays of the near offset traces. Figure 3 includes a near trace from every shot for line A and figures 4-22 include near traces from every 4 th shot of lines B and 1. Because of the small scales of these displays, the amplitudes of the near traces are modulated to enhance visual clarity. Details of how we apply the amplitude modulation are given in Lee et al.(1988).

\section{REFERENCES}

Barry, K.M., Cavers, D.A., and Kneale, C.W., 1975, Recommended standards for digital tape formats: Geophysics, v. 40, p. $344-352$.

Grow, J.A., and Markl, R.G., 1977, IPOD-USGS multichannel seismic reflection profile from Cape Hatteras to the Mid-Atlantic Ridge: Geology, v. 5, p. 625-630.

Lee, M.W., Agena, W.F., and Hutchinson, D.R., 1988, Processing of GLIMPCE multichannel Seismic Data: U.S. Geological Survey Open-File Report 88-225, 46 p. 


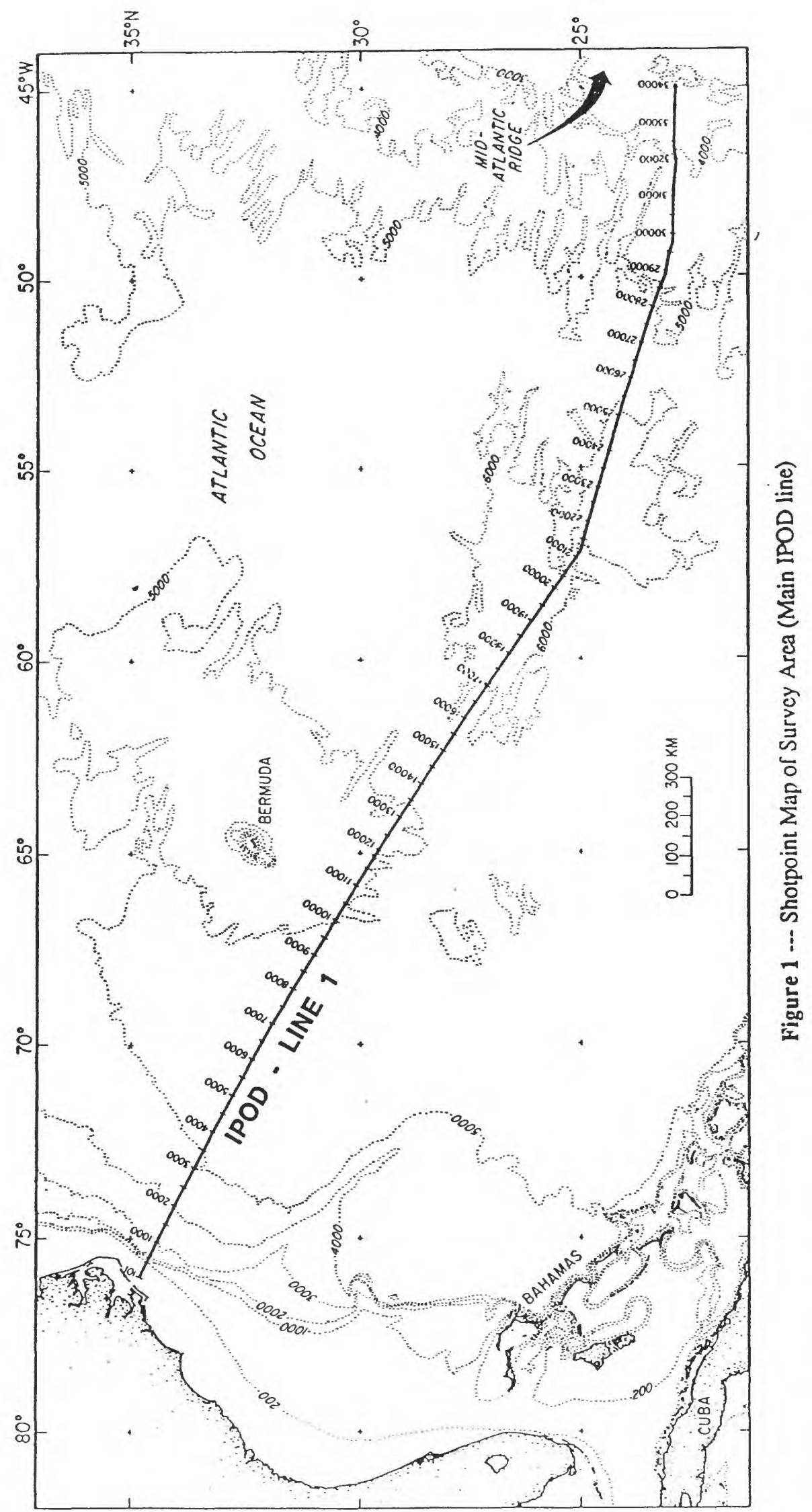




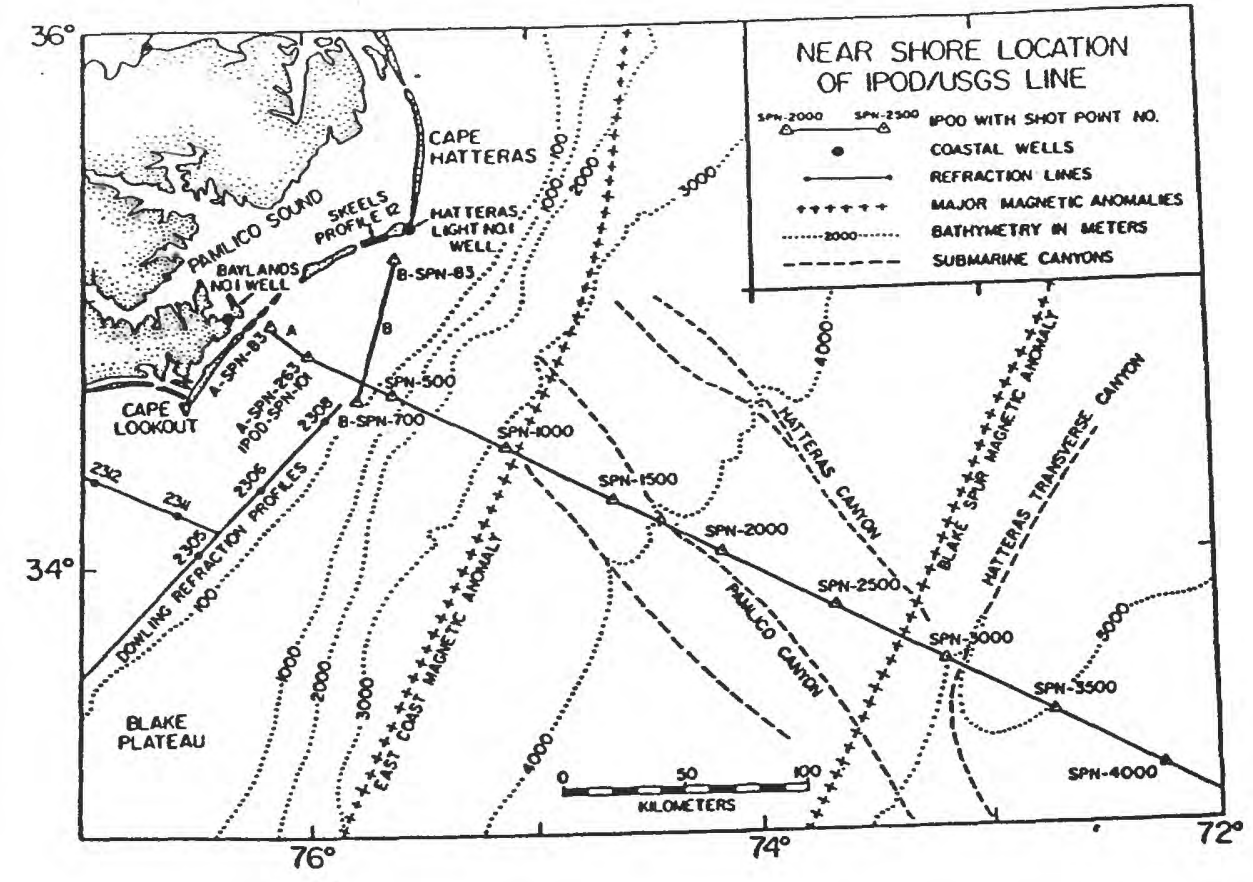

Figure 2 -- Shotpoint Map of IPOD close to Cape Hatteras showing lines A and B shot to tie the main IPOD line closer to wells (From Grow and Markl, 1977).

\begin{tabular}{|c|l|}
\hline DFS-III & Instrument \\
\hline $346 \mathrm{~m}$ & Near Offset Distance \\
\hline $50 \mathrm{~m}$ & Spacing between center of traces 48 through 25 \\
\hline $75 \mathrm{~m}$ & Spacing between center of trace 25 and trace 24 \\
\hline $100 \mathrm{~m}$ & Spacing between center of traces 24 through 1 \\
\hline $4 \mathrm{~ms}$ & Sample Rate \\
\hline $10-12 \mathrm{~s}$ & Recording length \\
\hline 36 & Fold Coverage \\
\hline $9 \mathrm{~m}$ & Gun Depth \\
\hline $1700 \mathrm{in} .^{3}$ & Displacement of Airgun Array \\
\hline $8-62 \mathrm{~Hz}$ & Recording Filter (with slopes of 18dB/octave) \\
\hline
\end{tabular}

Table 1 -- Field parameters used in survey 


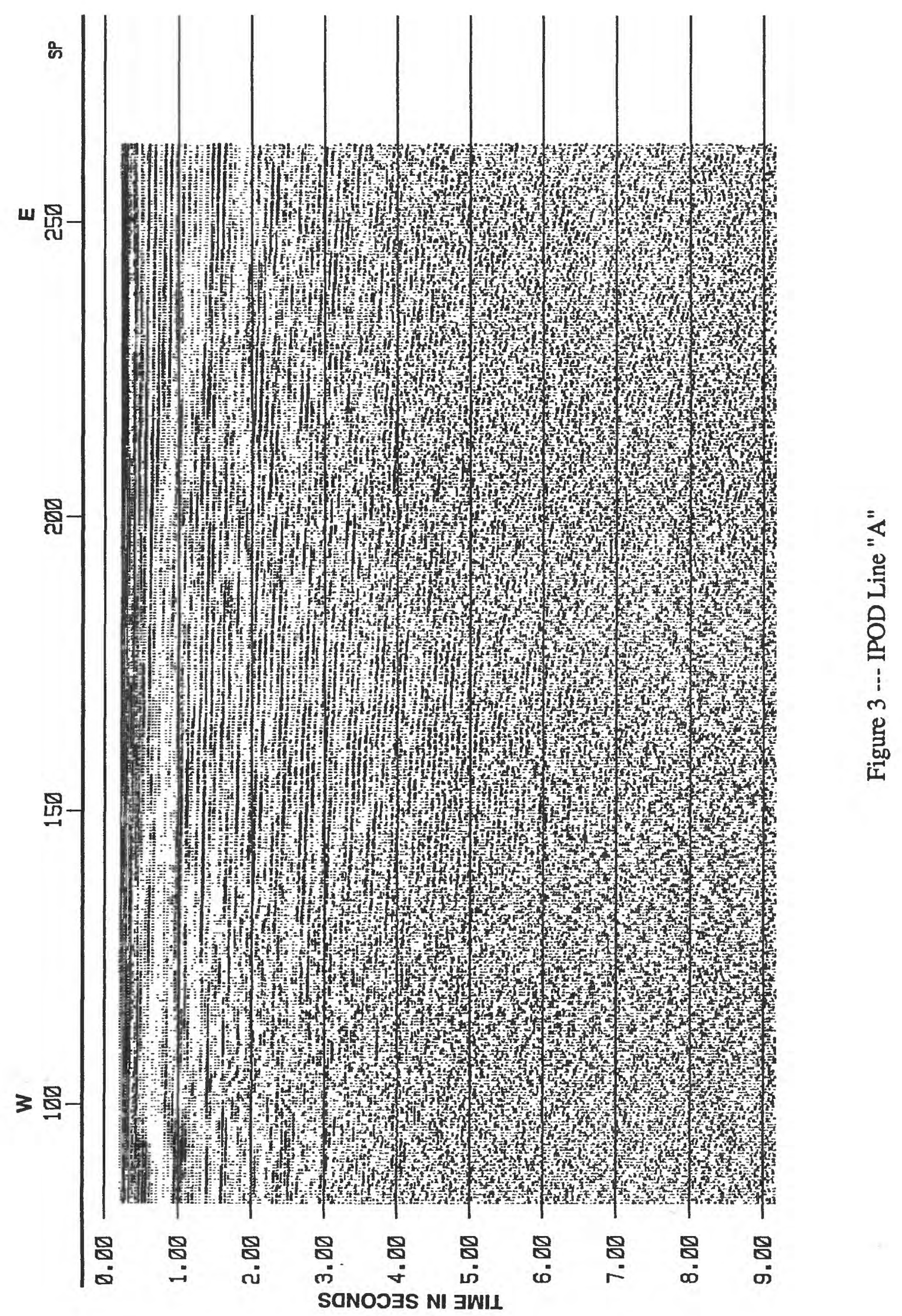




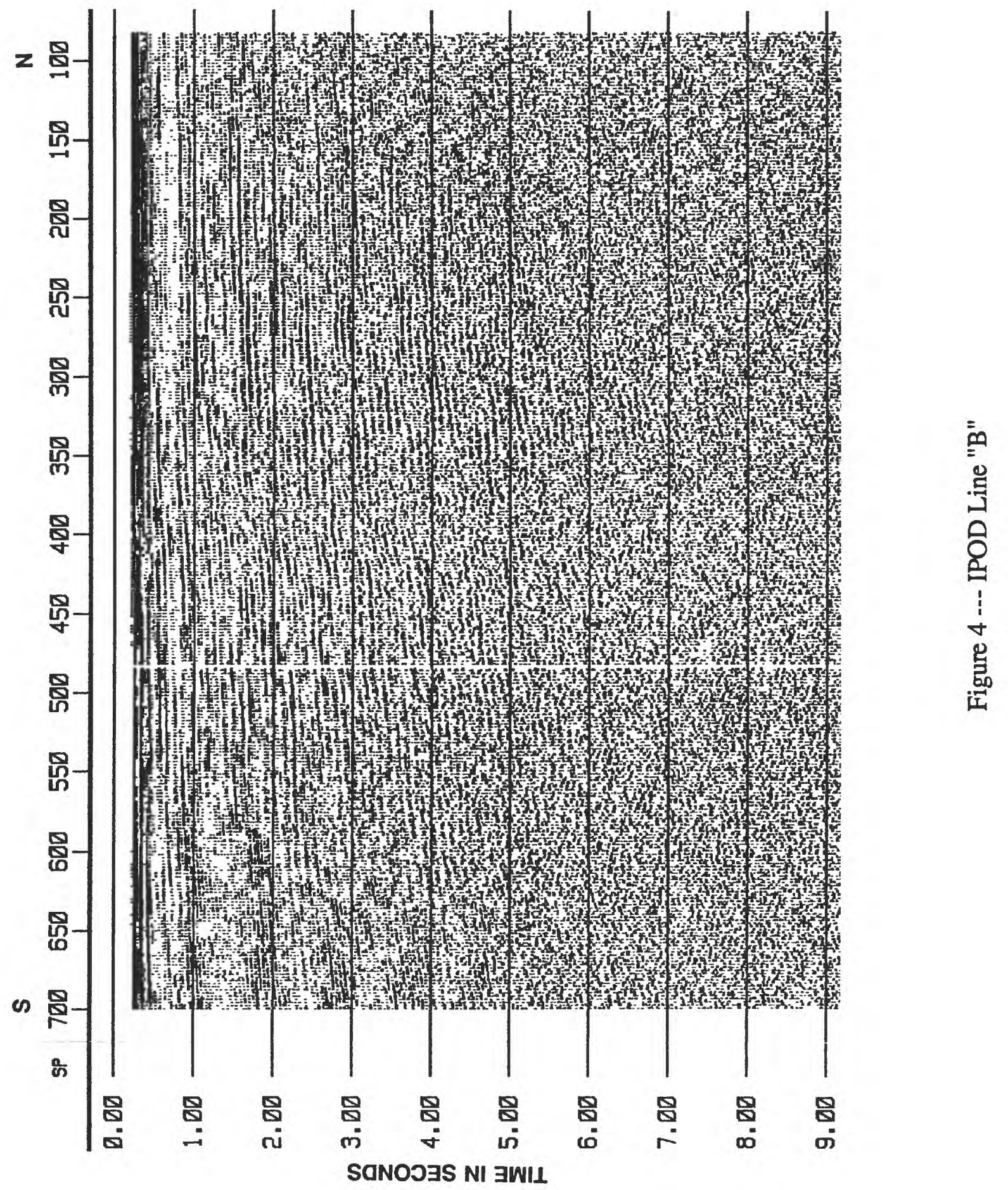




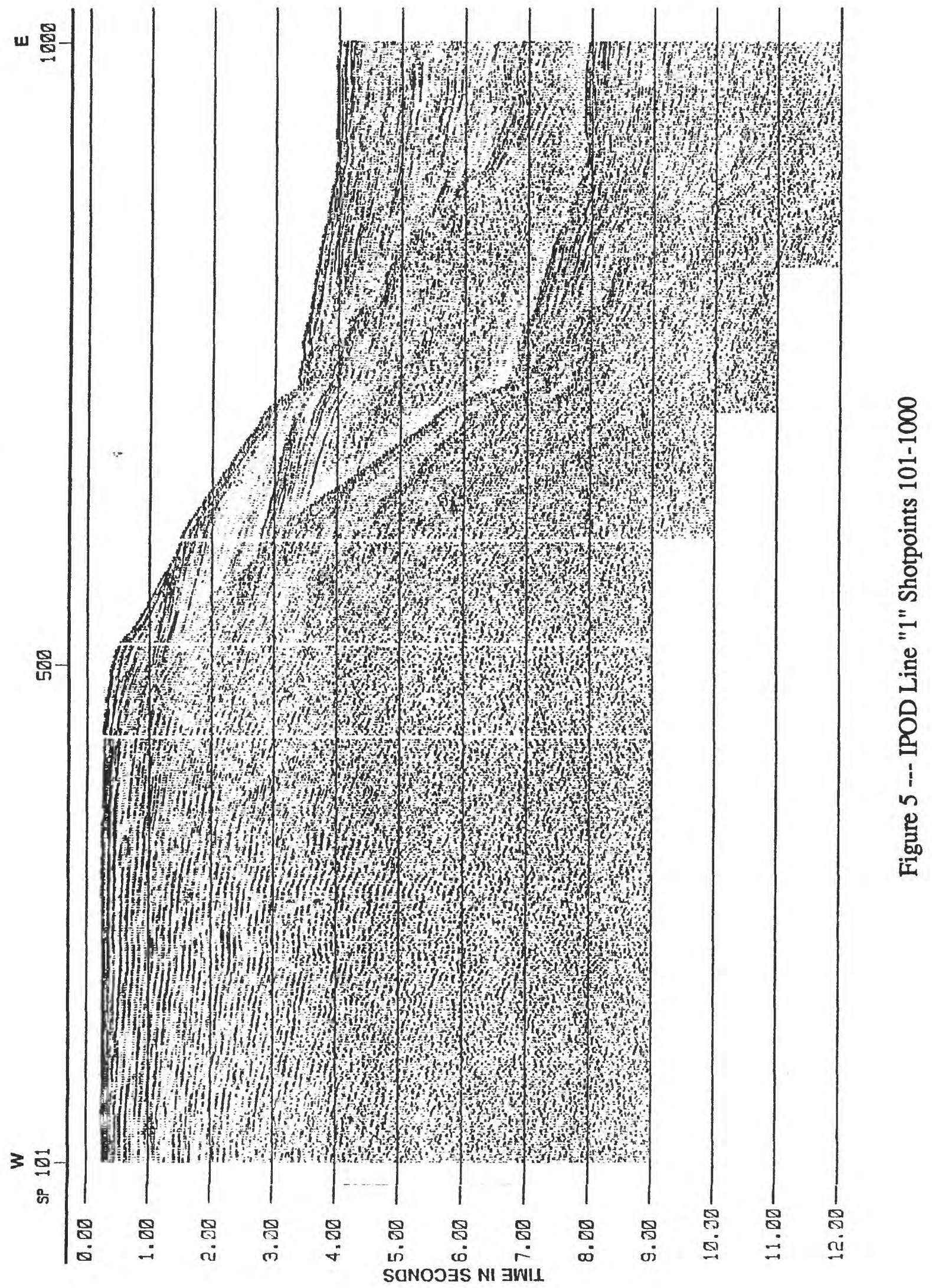




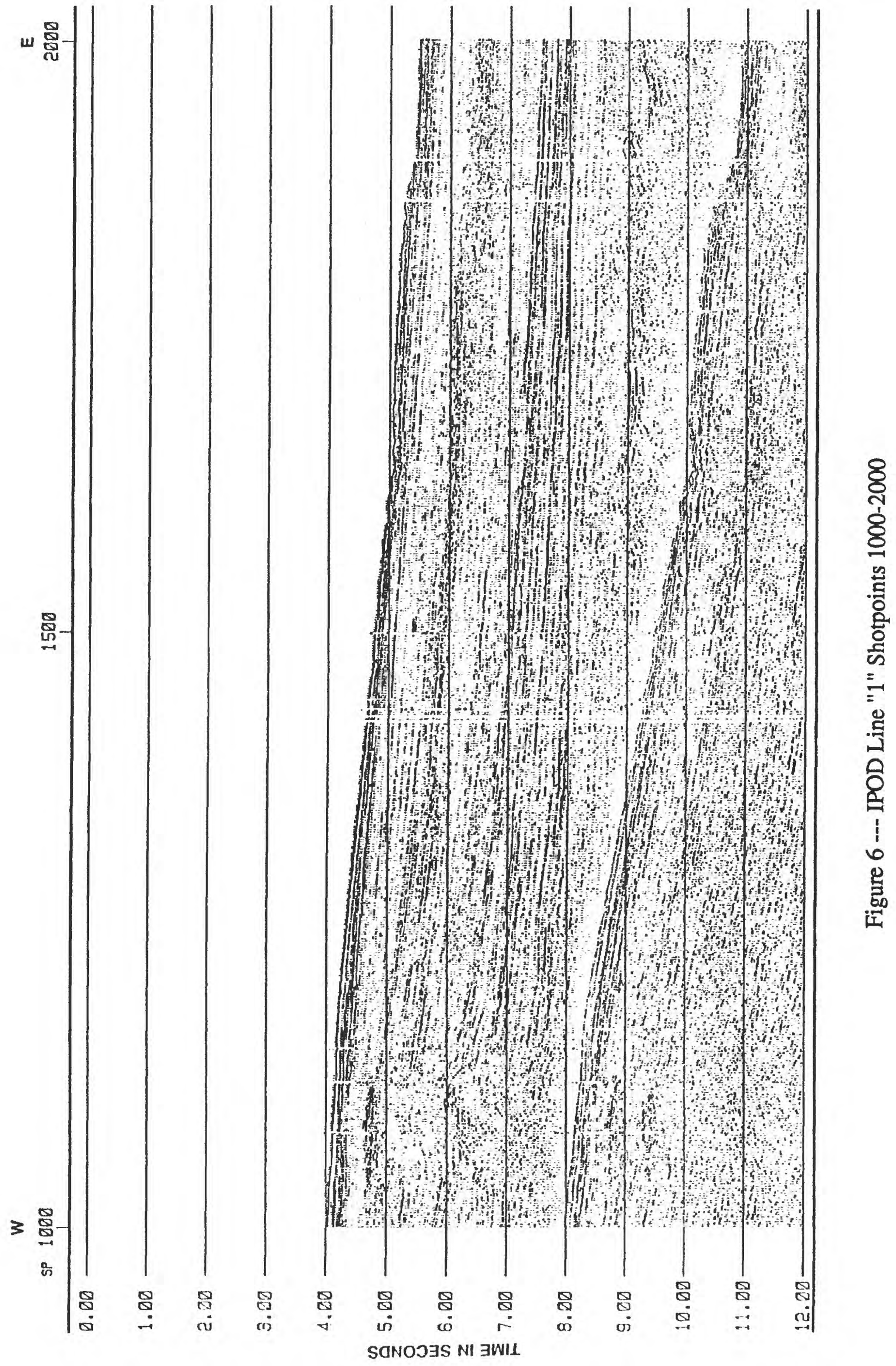


w 윰

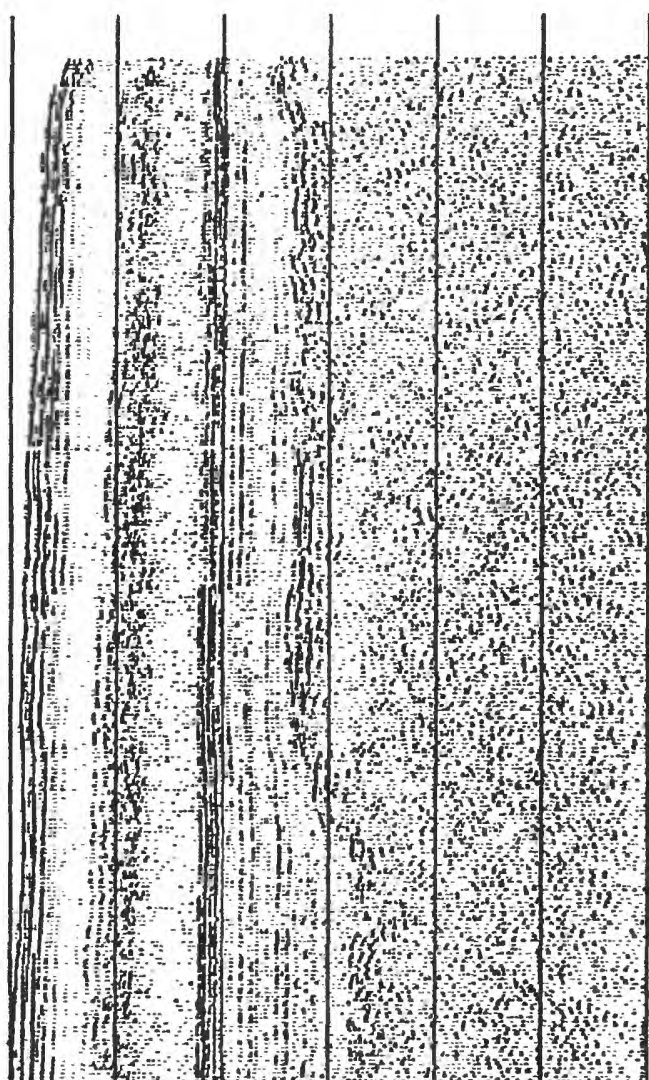

只

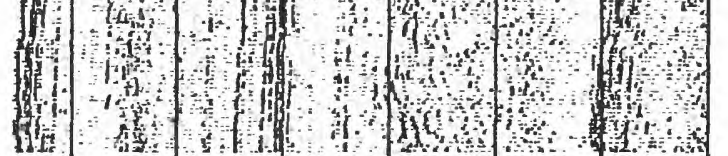

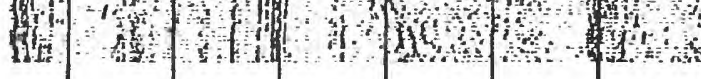

is

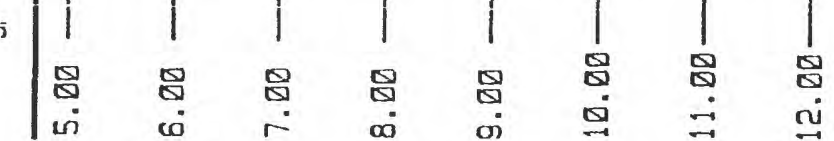
40 (I) 4 mom

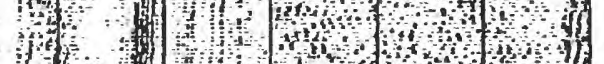

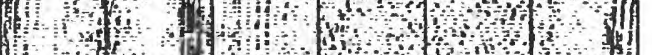

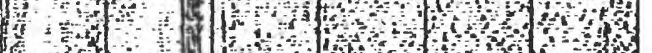

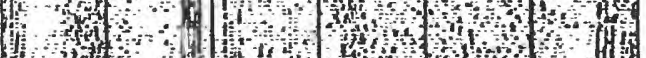
H. Wham

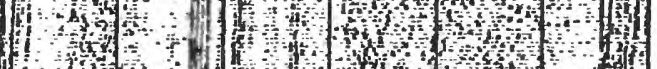
(4) (1) 4 w Hn 1 mow (m) mW

(I)

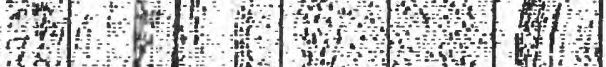
40 moln (1)
曹-

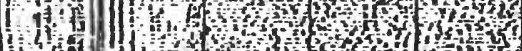
$14 m$ mons bow

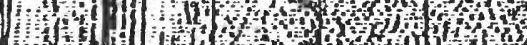

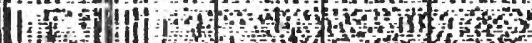
cy

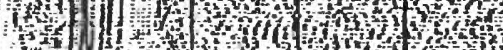
Hin

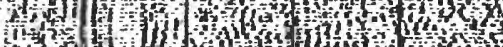

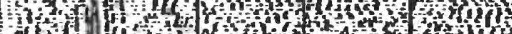

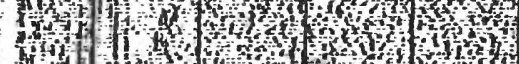

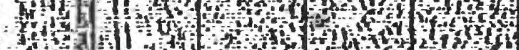

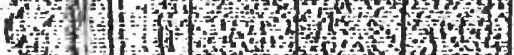

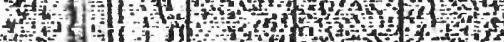
, f (t) th wh wh WW

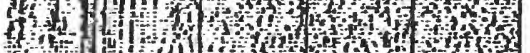

品-

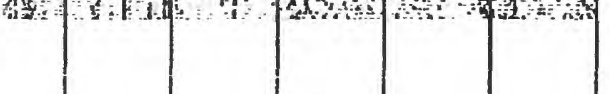




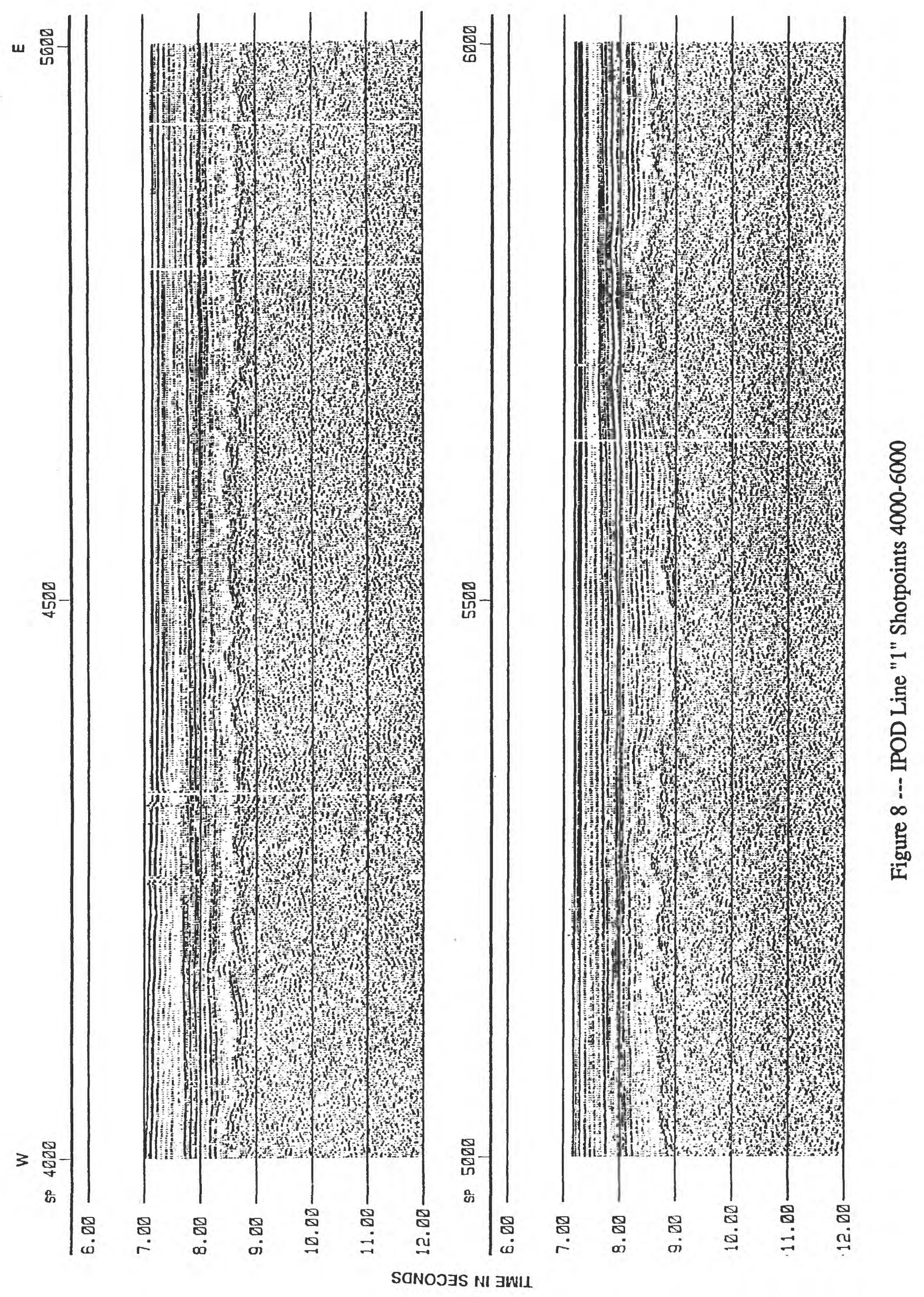




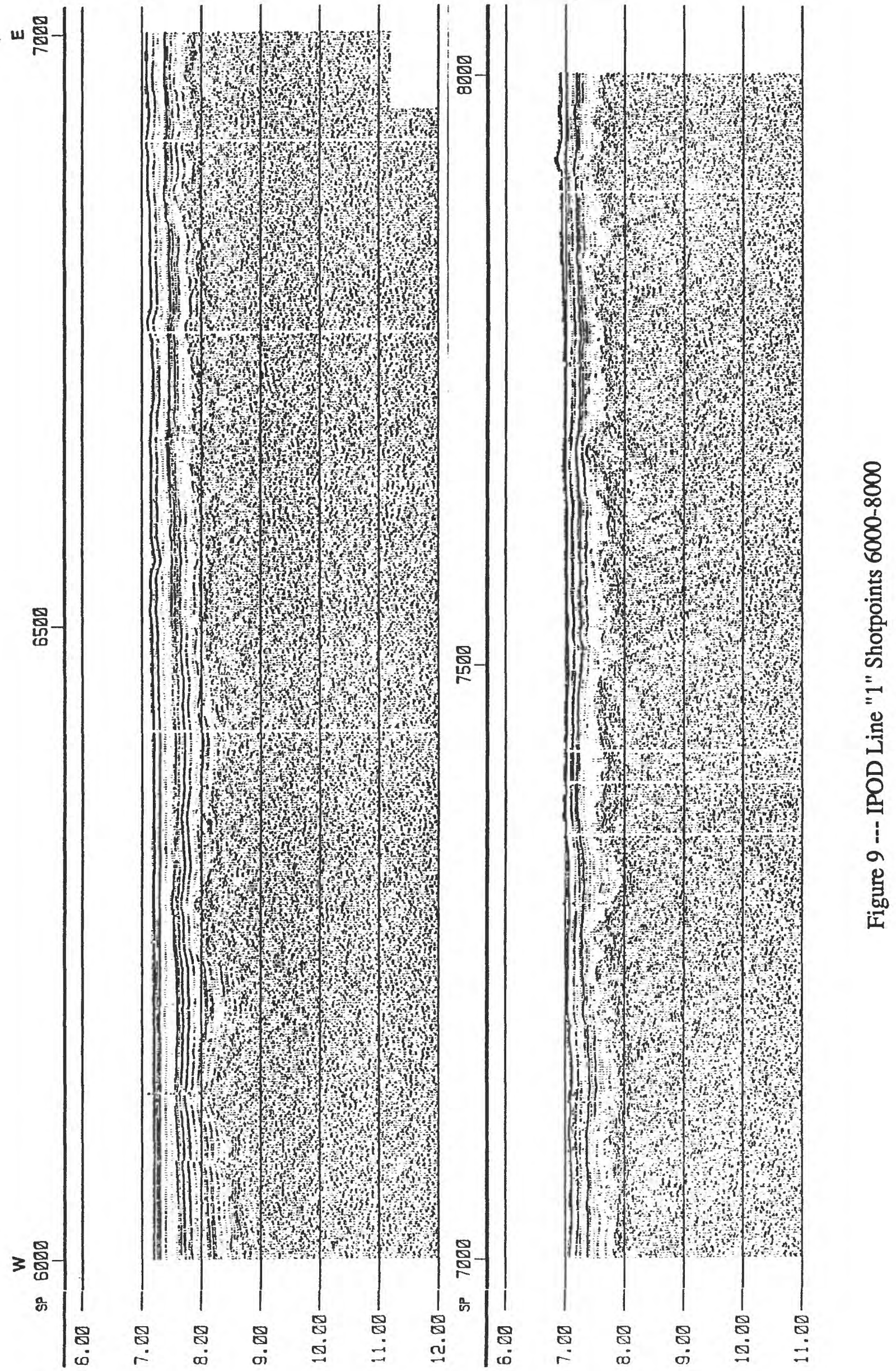




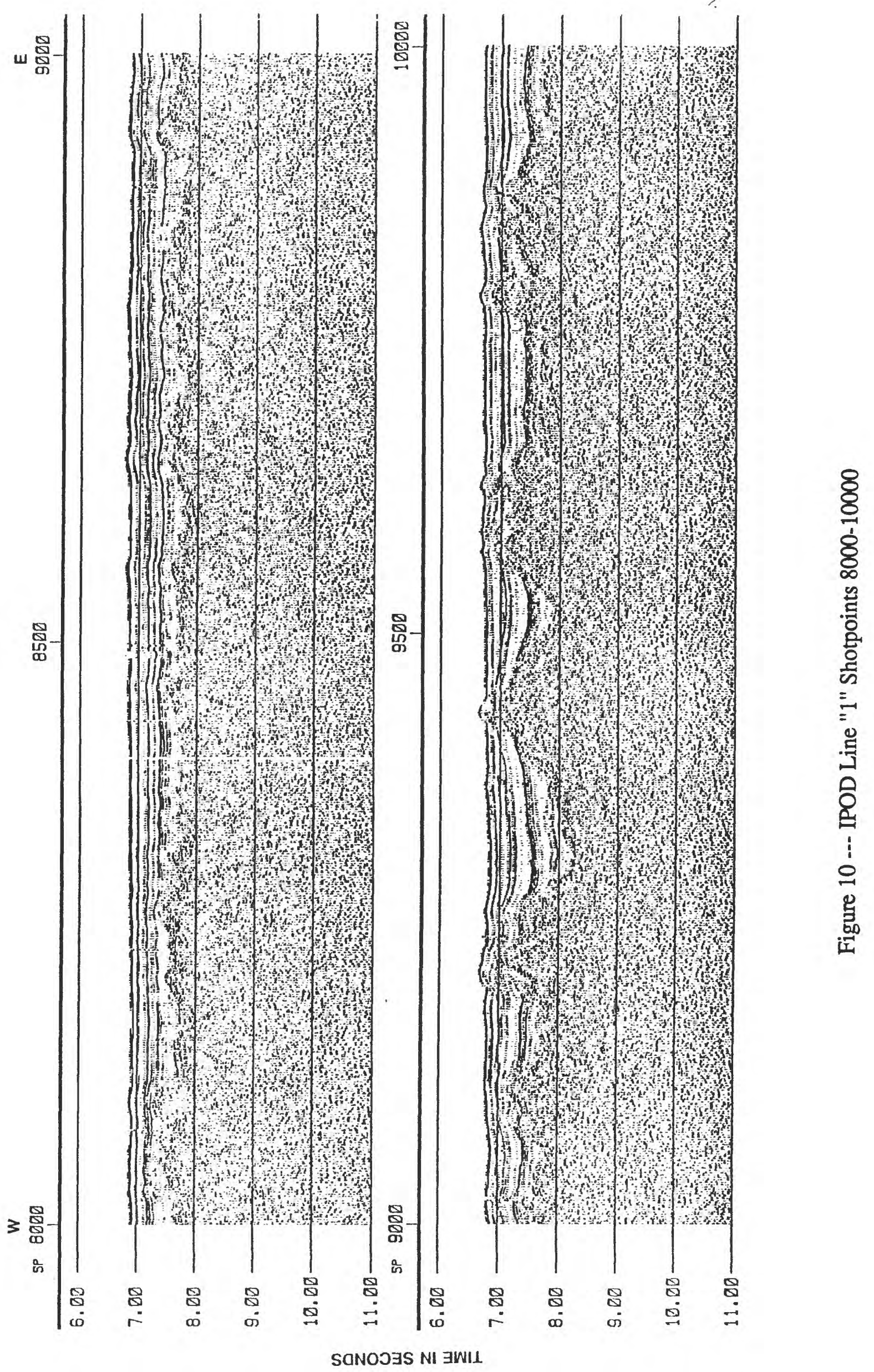




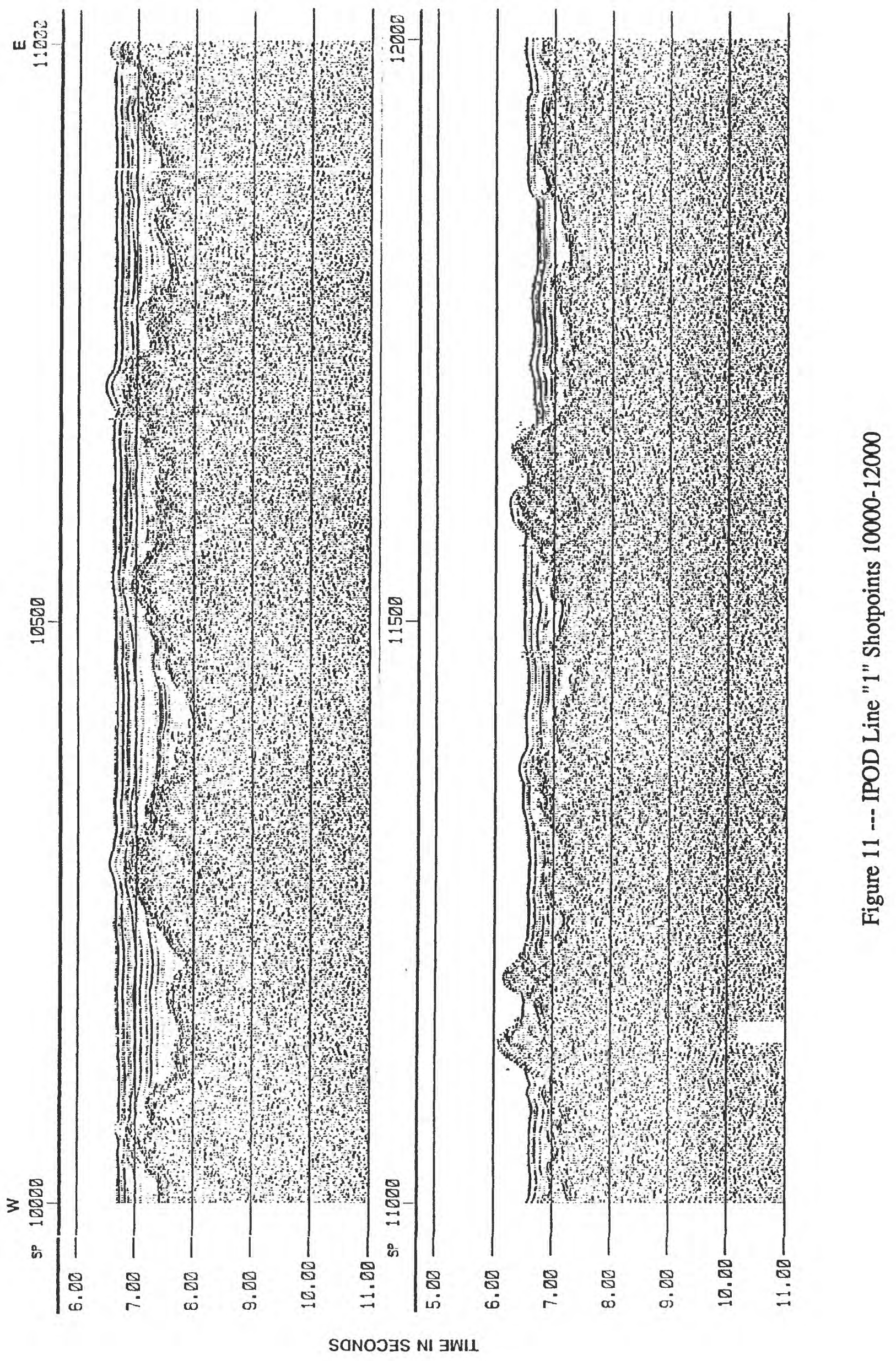




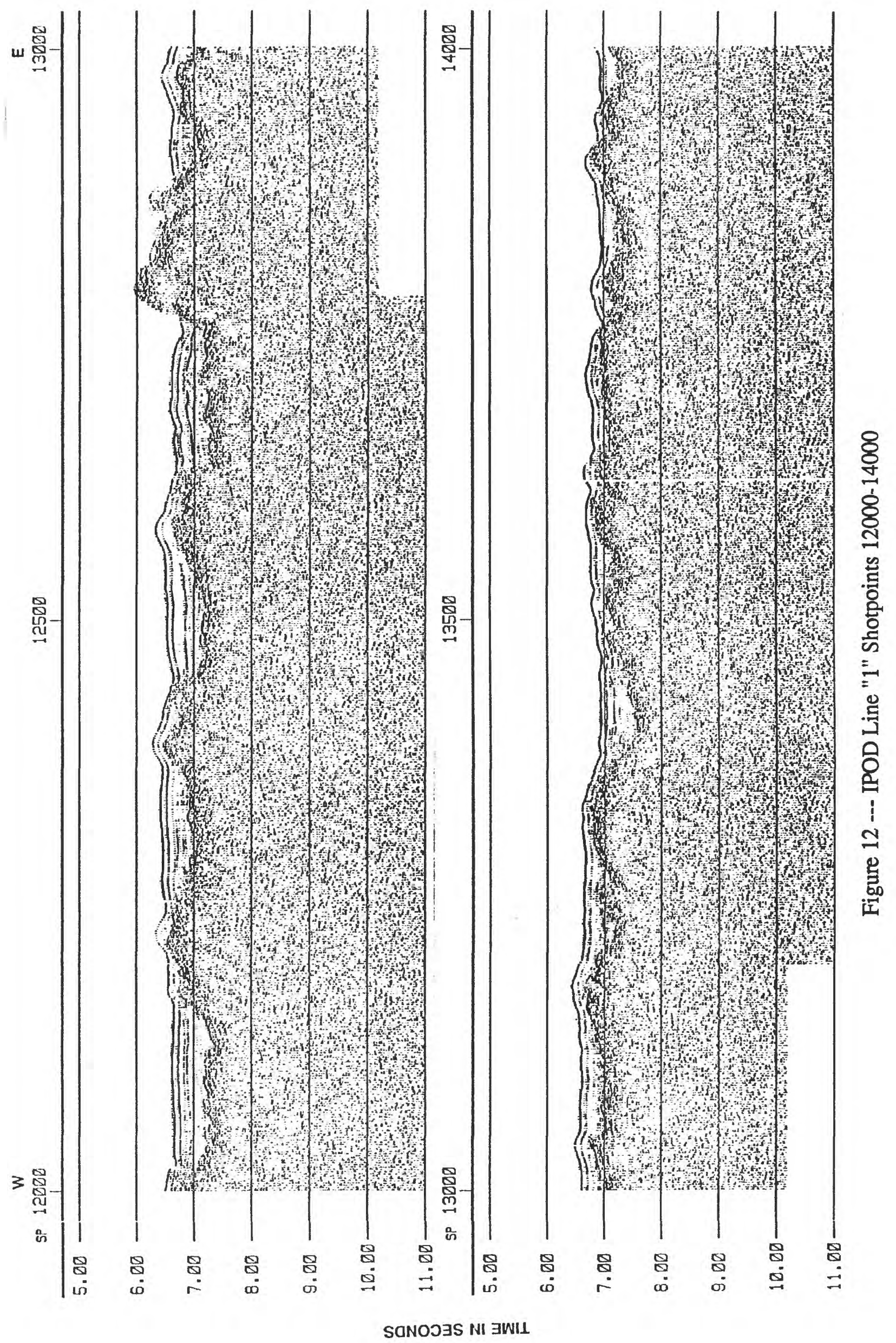




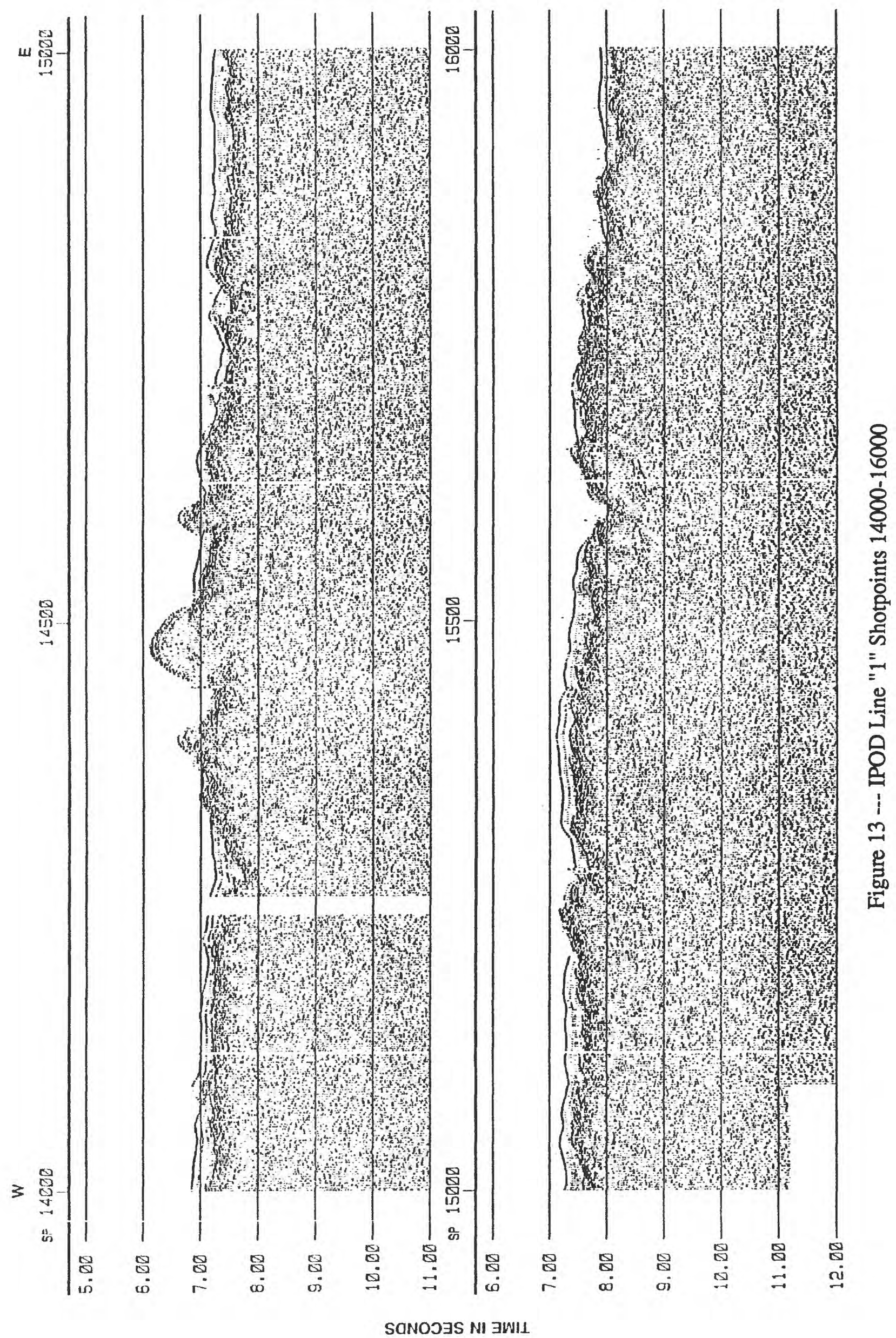




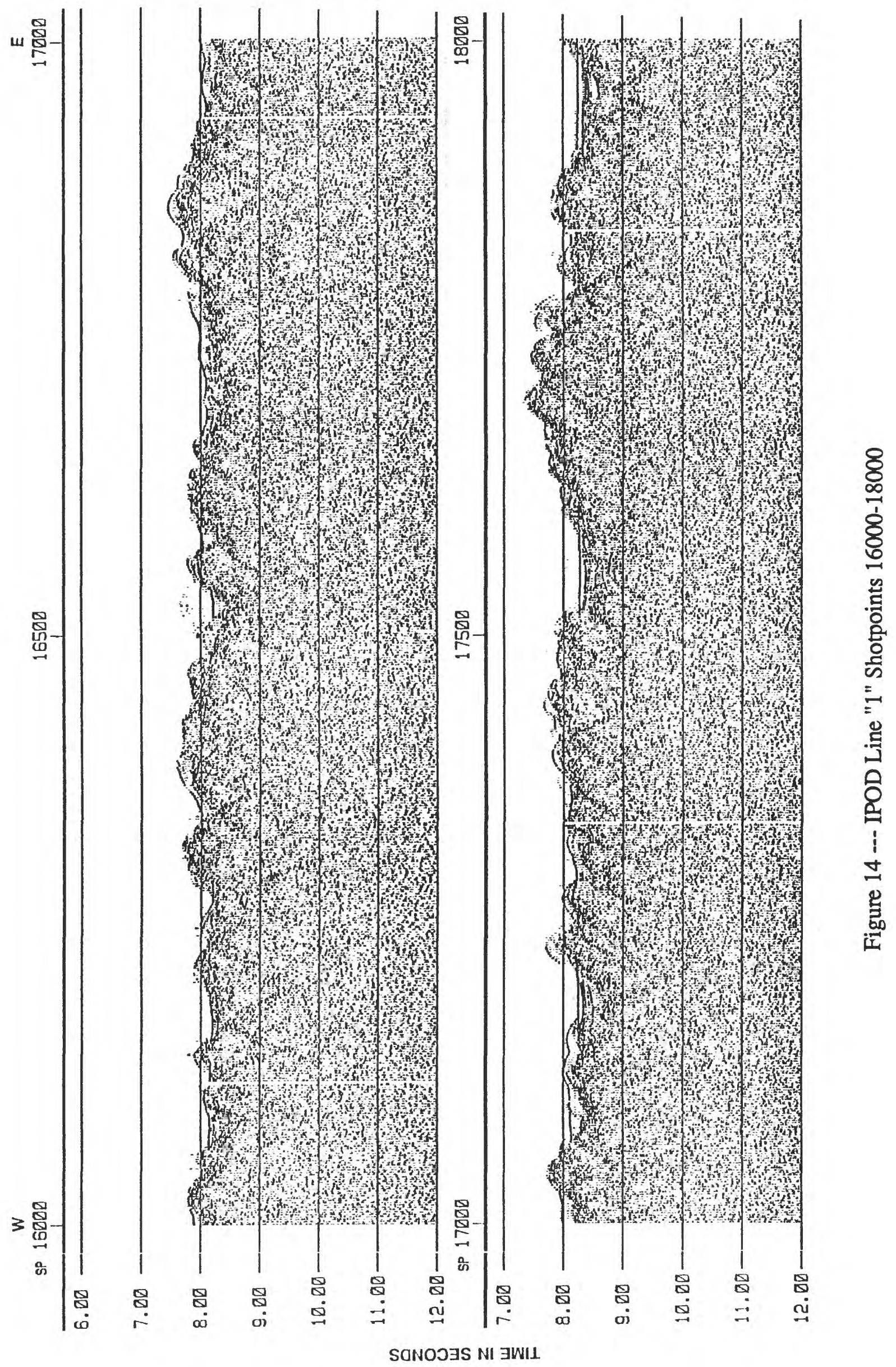




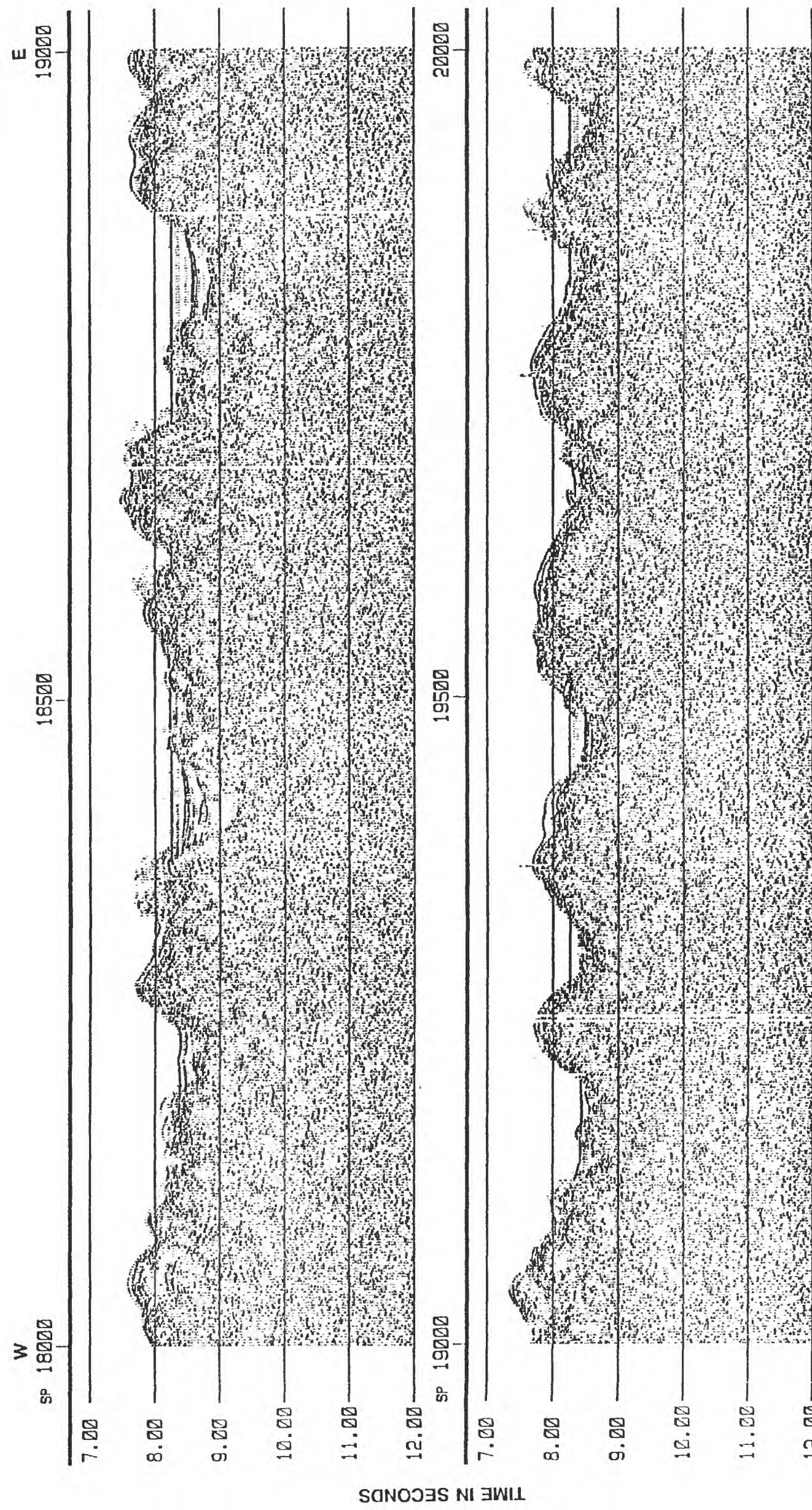

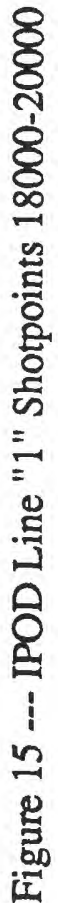




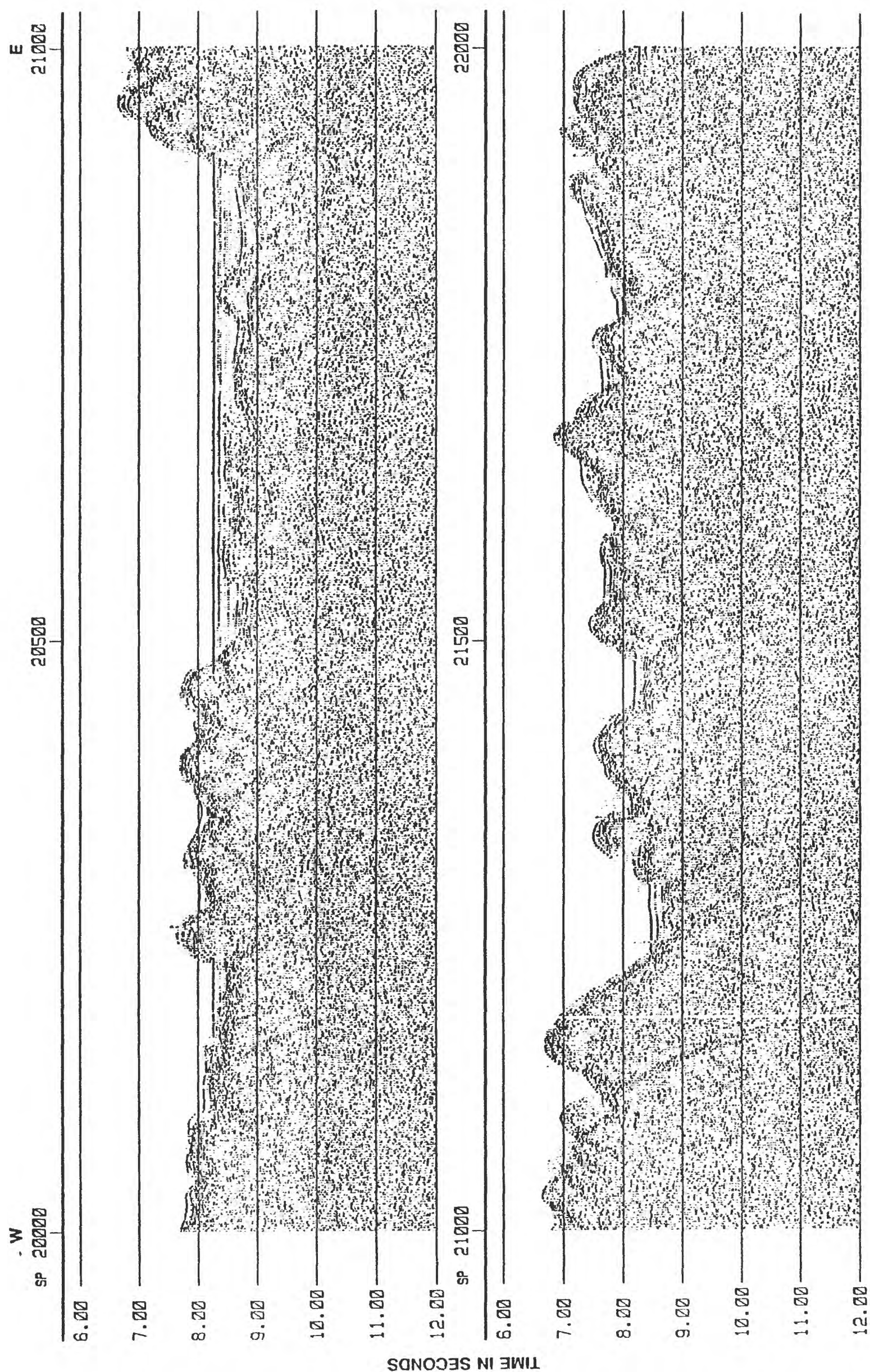

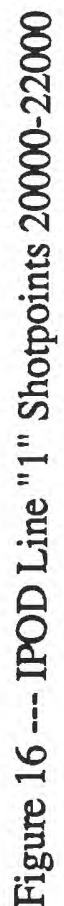




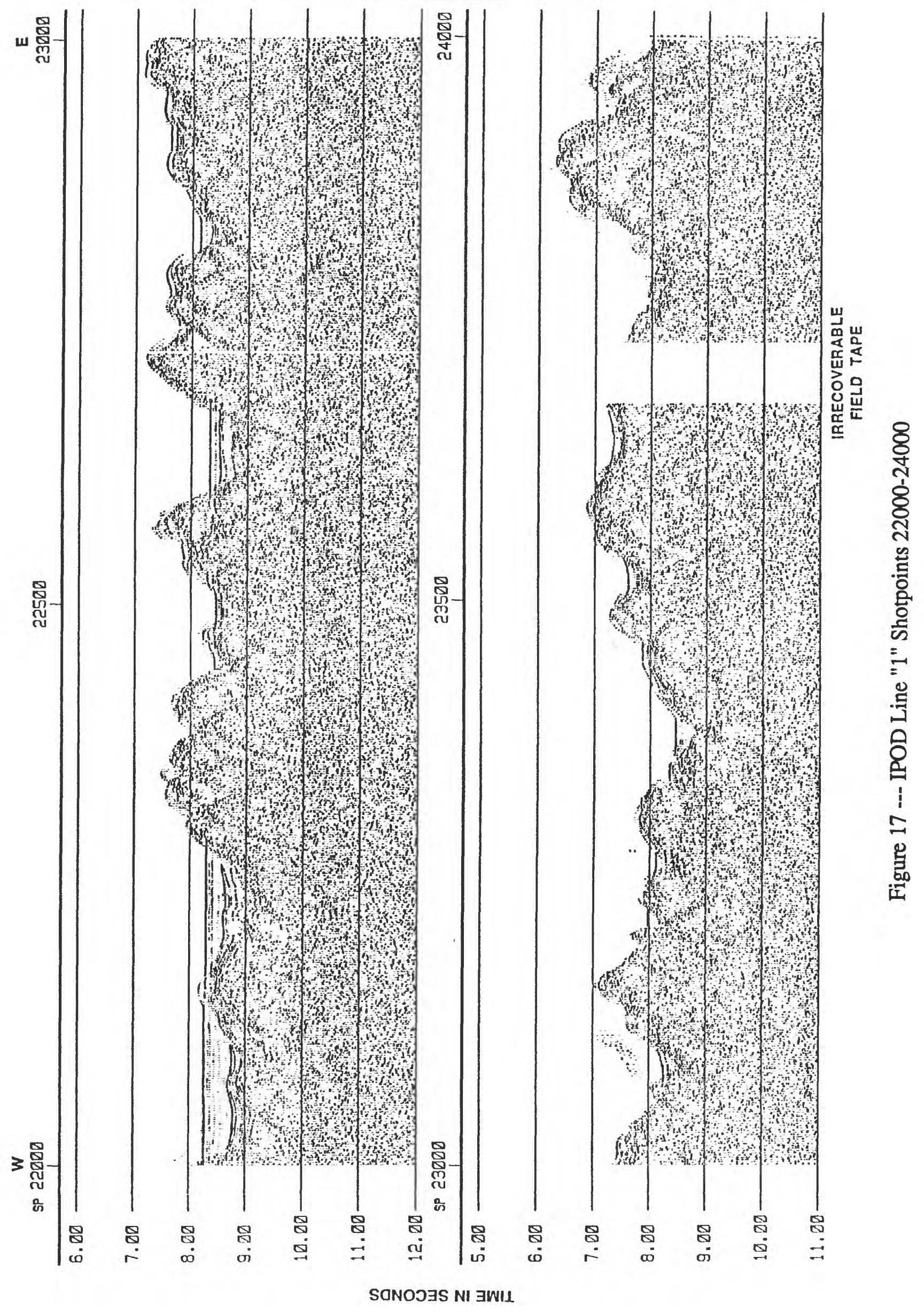




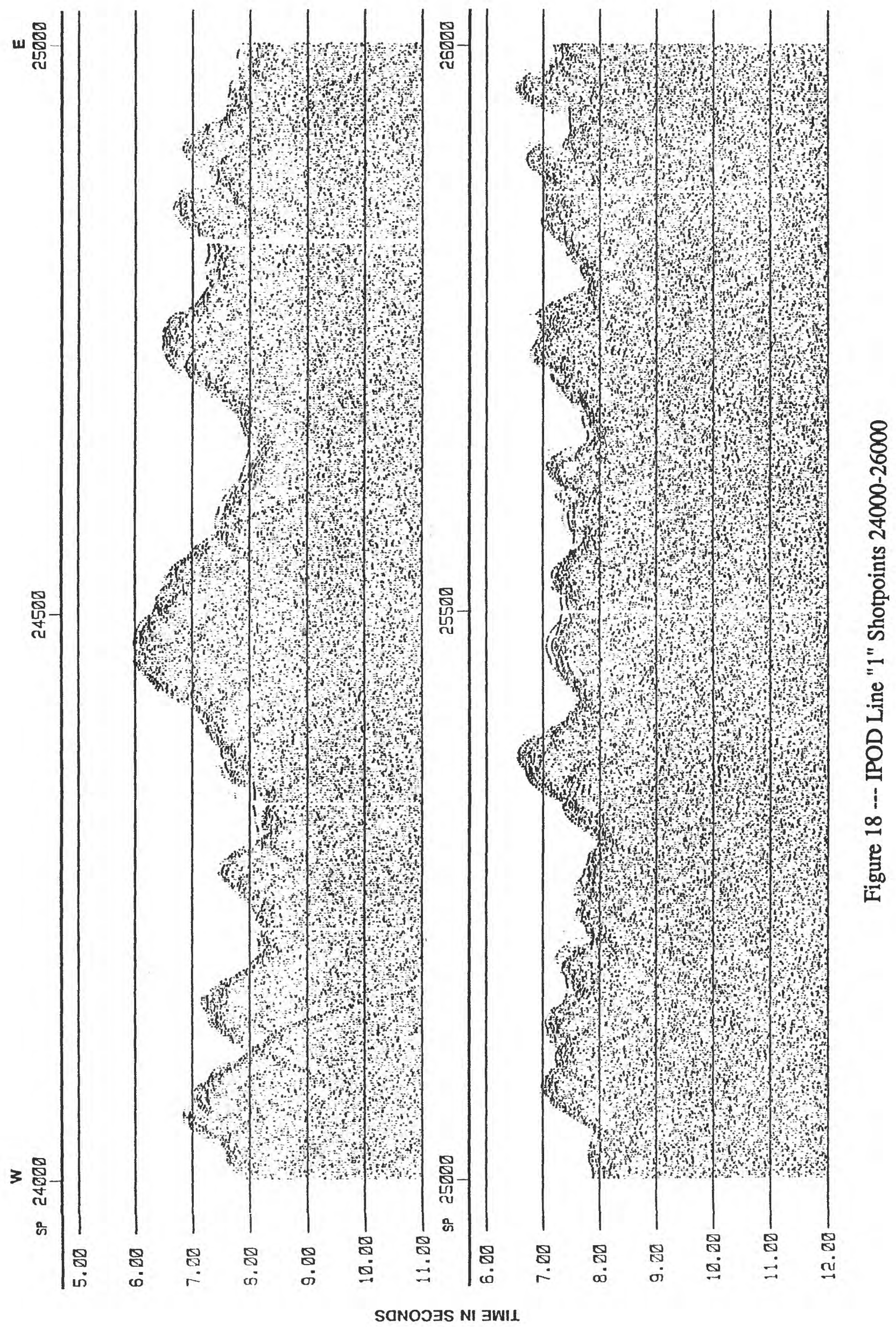




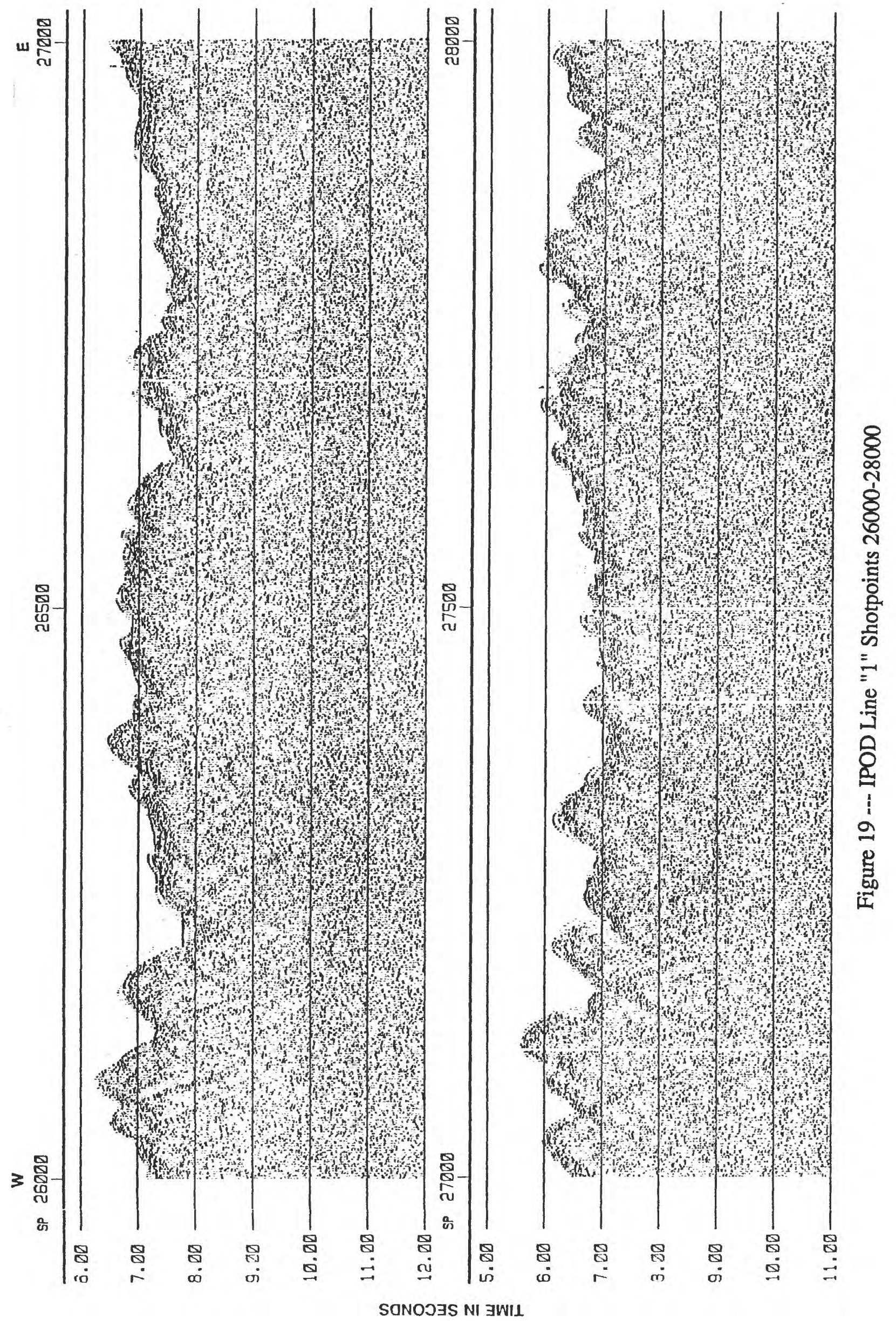


w ํํำ $1+1+1+1$ ช

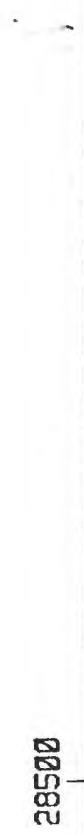

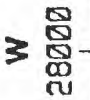

\&

|

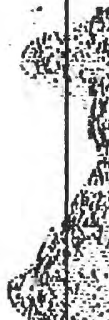

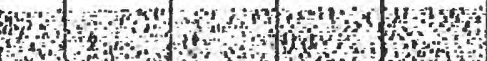
rom $1 \mathrm{mom}$ m

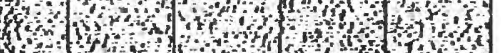

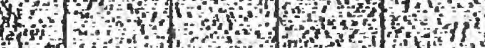

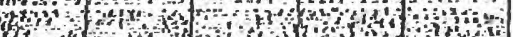

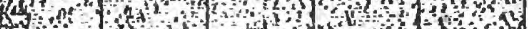
H m w m m

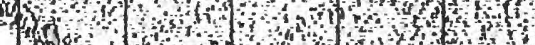
and $4 \mathrm{n}+\mathrm{H}+\mathrm{H}$ 2.

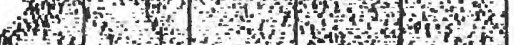

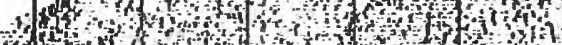

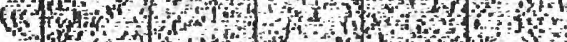
(x) whoth w w w

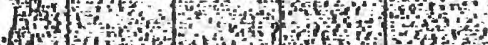
on (

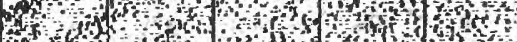

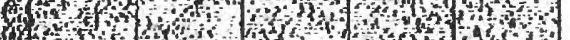
昌-1 \%

-
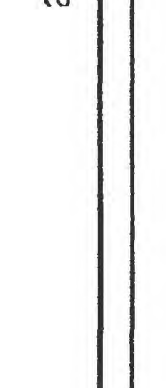

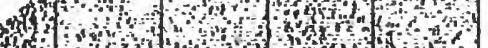
3 mat ind and to and 640

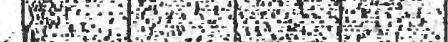
WhH $4 n^{2} m m^{2}$ Mn $\mathrm{mowh}_{\mathrm{m}}$

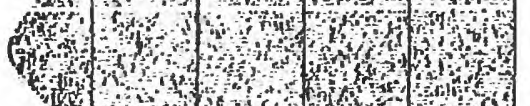
(n) (3)

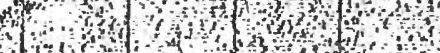
of

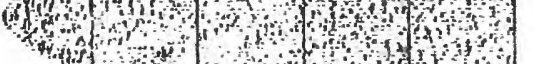
mon का का a st

昌

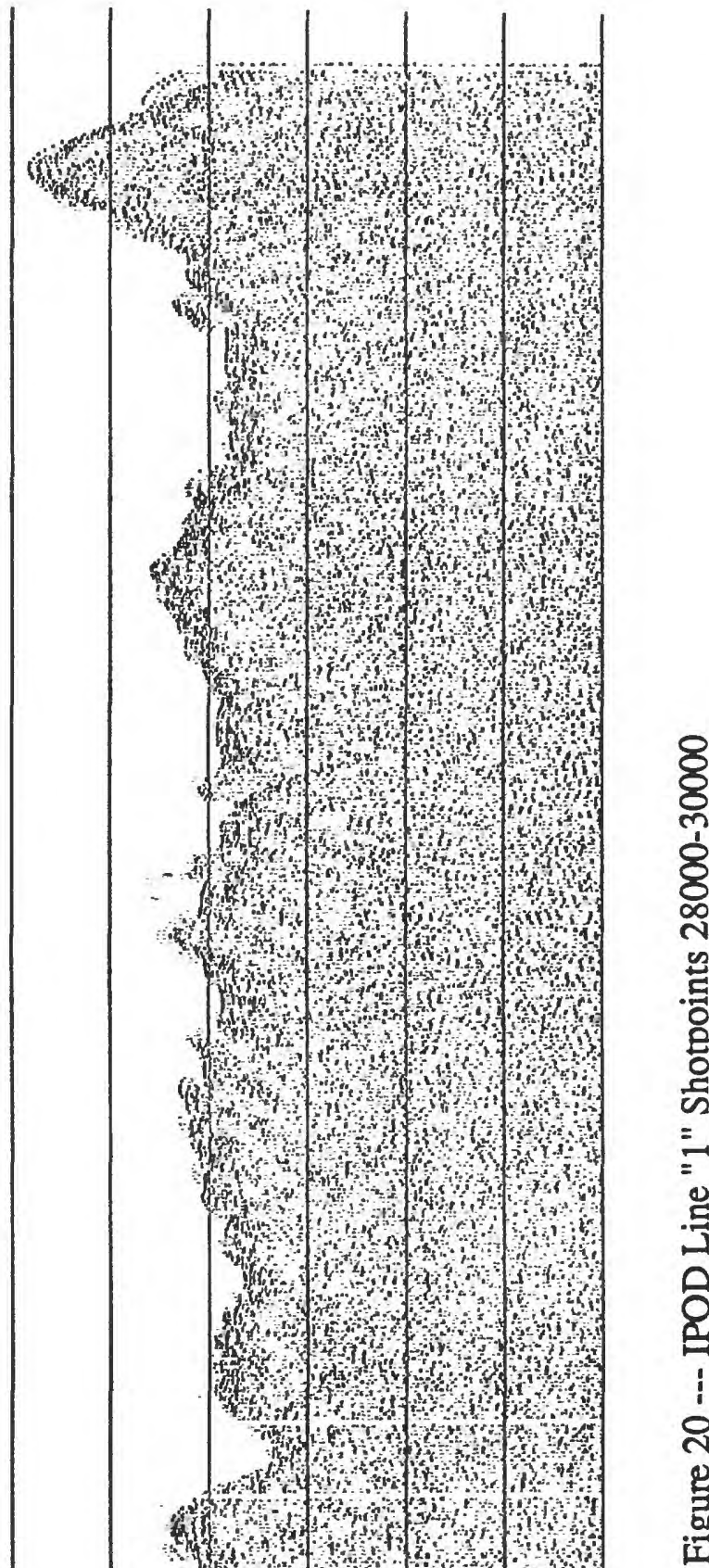

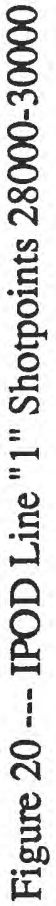




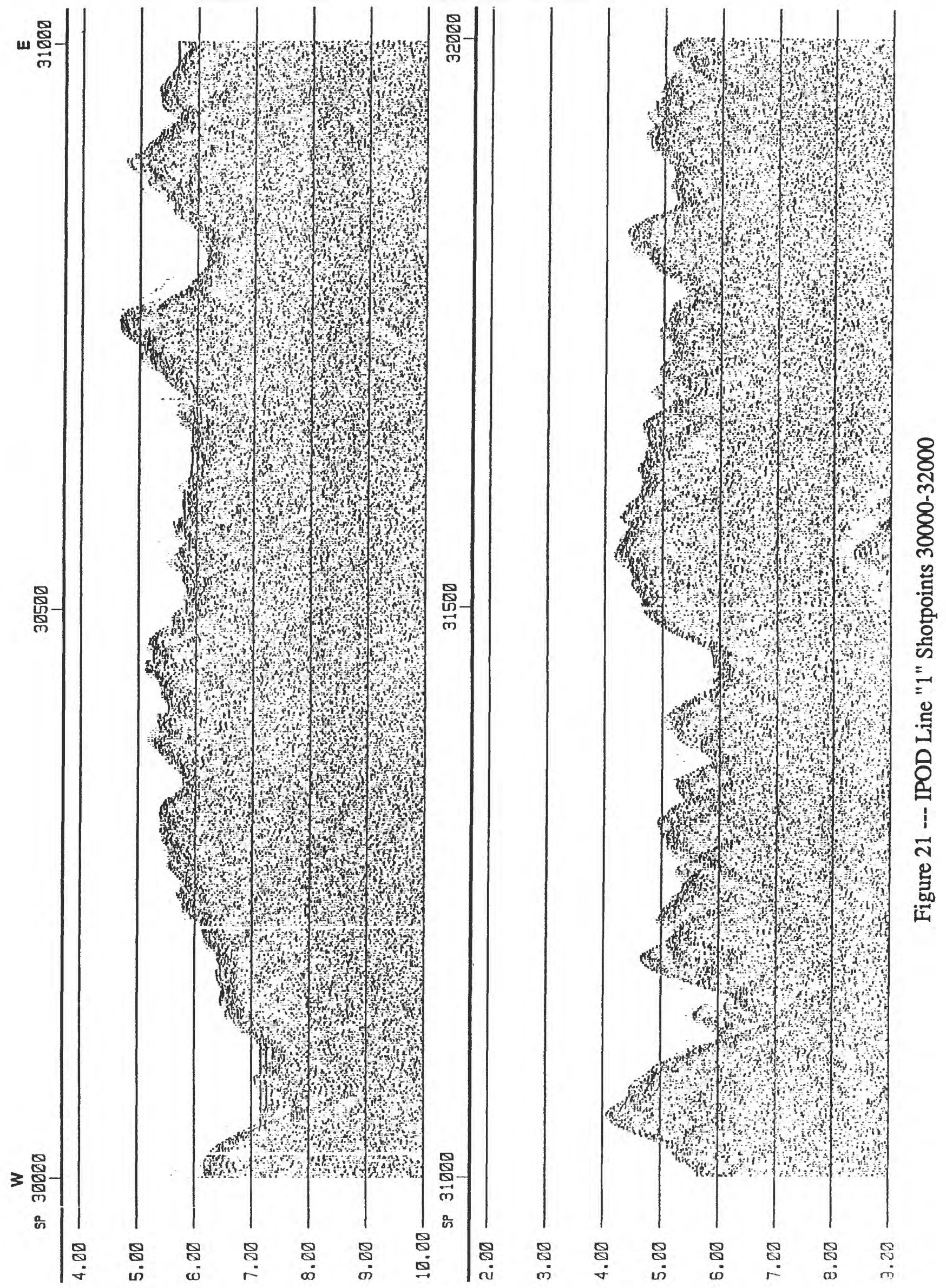




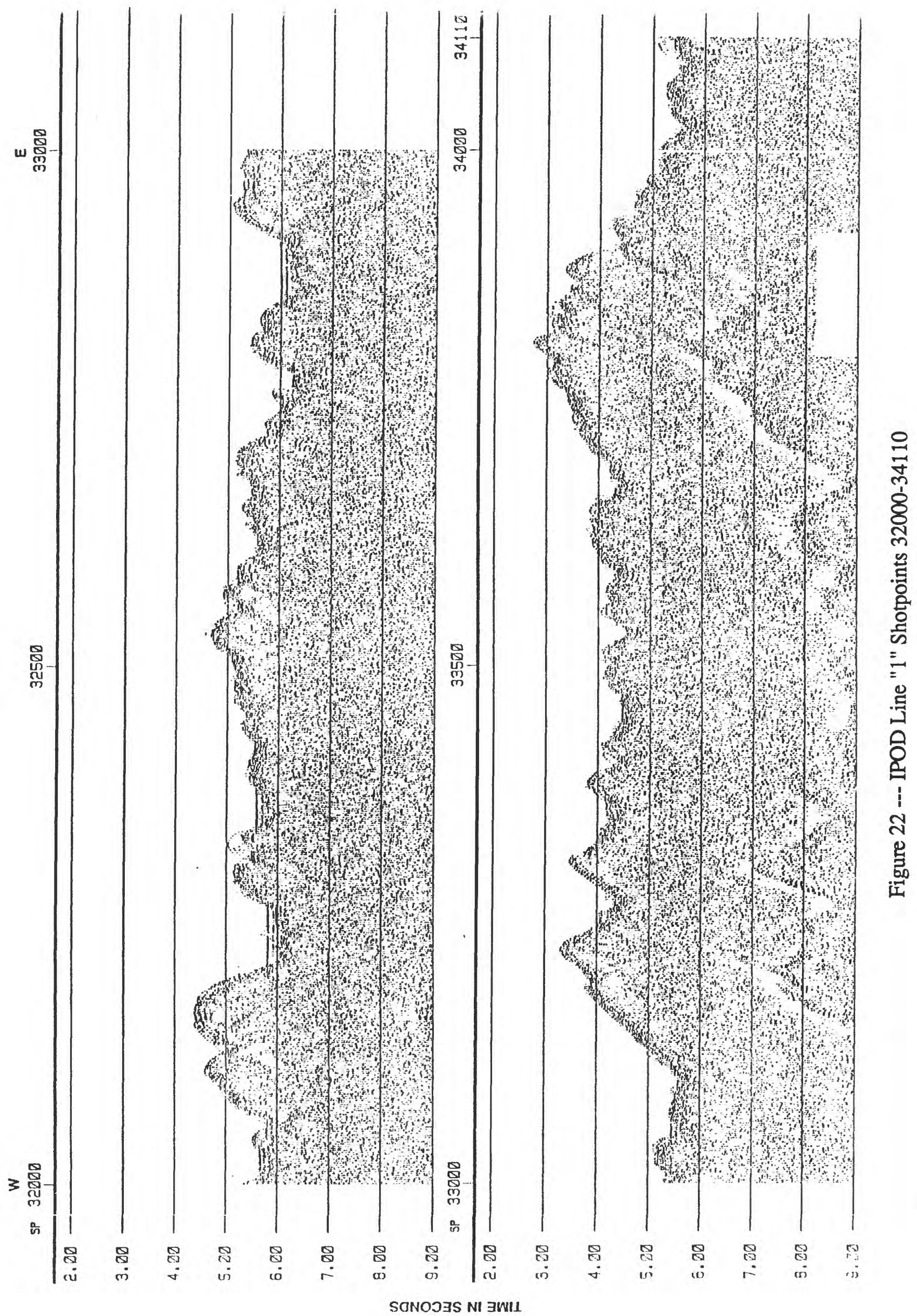


APPENDIX I

\begin{tabular}{|c|c|c|c|c|c|c|}
\hline SLOT & PROJECT & LINE & REEL & SPTS & FILES & TYPE \\
\hline $\begin{array}{l}15054 \\
15055\end{array}$ & $\begin{array}{l}\text { GULF SEAL } \\
\text { GULF SEAL }\end{array}$ & $\begin{array}{l}\text { IPOD-A } \\
\text { IPOD-A }\end{array}$ & $\begin{array}{l}\text { A-1 } \\
\text { A-2 }\end{array}$ & $\begin{array}{c}263-135 \\
135-83\end{array}$ & $\begin{array}{c}1-256 \\
257-362\end{array}$ & $\begin{array}{l}\text { DEMUX } \\
\text { DEMUX }\end{array}$ \\
\hline $\begin{array}{l}8372 \\
8373 \\
8374 \\
8375 \\
8376 \\
8377\end{array}$ & $\begin{array}{l}\text { GULF SEAL } \\
\text { GULF SEAL } \\
\text { GULF SEAL } \\
\text { GULF SEAL } \\
\text { GULF SEAL } \\
\text { GULF SEAL }\end{array}$ & $\begin{array}{l}\text { IPOD-B } \\
\text { IPOD-B } \\
\text { IPOD-B } \\
\text { IPOD-B } \\
\text { IPOD-B } \\
\text { IPOD-B }\end{array}$ & $\begin{array}{l}\text { B-1 } \\
\text { B-2 } \\
\text { B-3 } \\
\text { B-4 } \\
\text { B-5 } \\
\text { B-6 }\end{array}$ & $\begin{array}{c}700-578 \\
577-453 \\
454-329 \\
329-203 \\
203-151 \\
151-83\end{array}$ & $\begin{array}{c}1-246 \\
246-495 \\
494-743 \\
743-996 \\
996-99 \\
99-235\end{array}$ & $\begin{array}{l}\text { DEMUX } \\
\text { DEMUX } \\
\text { DEMUX } \\
\text { DEMUX } \\
\text { DEMUX } \\
\text { DEMUX }\end{array}$ \\
\hline 4567 & GULF SEAL & & & & & \\
\hline & & IPUD & IPODIA & $101-228$ & $1-256$ & DEMUX \\
\hline $\begin{array}{l}4566 \\
6453\end{array}$ & $\begin{array}{l}\text { GULF SEAL } \\
\text { GULF SEAL }\end{array}$ & IPOD & IPOD1B & $228-422$ & $256-643$ & DEMUX \\
\hline 4565 & $\begin{array}{l}\text { GULF SEAL } \\
\text { GULF SEAL }\end{array}$ & IPOD & IPOD1C & $422-549$ & $643-897$ & DEMUX \\
\hline 13244 & $\begin{array}{l}\text { GULF SEAL } \\
\text { GULF SEAL }\end{array}$ & $\begin{array}{l}\text { IPOD } \\
\text { IPOD }\end{array}$ & $\begin{array}{l}\text { IPODID } \\
\text { IPOD-1 }\end{array}$ & $\begin{array}{l}549-600 \\
600-727\end{array}$ & $\begin{array}{l}897-999 \\
999-254\end{array}$ & $\begin{array}{l}\text { DEMUX } \\
\text { DEMUX }\end{array}$ \\
\hline 13245 & GULF SEAL & IPOD & IPOD-2 & $727-854$ & 254-508 & $\begin{array}{l}\text { DEMUX } \\
\text { DEMUX }\end{array}$ \\
\hline 13246 & GULF SEAL & IPOD & IPOD-3 & $854-984$ & $508-768$ & DEMUX \\
\hline 13247 & GULF SEAL & IPOD & IPOD-4 & $984-1102$ & $768-4$ & DEMUX \\
\hline 13248 & GULF SEAL & IPOD & IPOD-5 & $1102-1229$ & $5-258$ & DEMUX \\
\hline 13249 & GULF SEAL & IPOD & IPOD-6 & $1229-1356$ & $258-512$ & DEMUX \\
\hline 13250 & GULF SEAL & IPOD & IPOD-7 & $1356-1482$ & $512-764$ & DEMUX \\
\hline 13251 & GULF SEAL & IPOD & IPOD-8 & $1482-1575$ & $764-950$ & DEMUX \\
\hline 13252 & GULF SEAL & IPOD & IPOD-9 & $1539 \mathrm{~A}-1668$ & $1-260$ & DEMUX \\
\hline 13253 & GULF SEAL & IPOD & IPOD-10 & $1668-1795$ & $260-513$ & DEMUX \\
\hline 13254 & GULF SEAL & IPOD & IPOD-11 & $1795-1921$ & $513-766$ & DEMUX \\
\hline 13255 & GULF SEAL & IPOD & IPOD-12 & $1921-2051$ & $766-26$ & DEMUX \\
\hline 13256 & GULF SEAL & IPOD & IPOD-13 & $2051-2067$ & $26-58$ & DEMUX \\
\hline 13257 & GULF SEAL & IPOD & IPOD-14 & $2068-2194$ & $59-312$ & DEMUX \\
\hline 13258 & GULF SEAL & IPOD & IPOD-15 & $2194-2321$ & $312-566$ & DEMUX \\
\hline 13259 & GULF SEAL & IPOD & IPOD-16 & $2321-2348 \mathrm{~A}$ & $566-98$ & DEMUX \\
\hline 13260 & GULF SEAL & IPOD & IPOD-17 & $2348 \mathrm{~A}-2478$ & $98-357$ & DEMUX \\
\hline 13261 & GULF SEAL & IPOD & IPOD-18 & $2478-2585$ & $357-572$ & DEMUX \\
\hline 13262 & GULF SEAL & IPOD & IPOD-19 & $2586-2740$ & $573-882$ & DEMUX \\
\hline 13263 & GULF SEAL & IPOD & IPOD-20 & $2740-2881$ & $882-164$ & DEMUX \\
\hline 13264 & GULF SEAL & IPOD & IPOD-21 & $2882-3057$ & $165-516$ & DEMUX \\
\hline 13265 & GULF SEAL & IPOD & IPOD-22 & $3057-3102$ & $516-606$ & DEMUX \\
\hline 13266 & GULF SEAL & IPOD & IPOD-23 & $3103-3274$ & $607-950$ & DEMUX \\
\hline 13267 & GULF SEAL & IPOD & IPOD-24 & $3274-3407$ & $950-336$ & DEMUX \\
\hline 13268 & GULF SEAL & IPOD & IPOD-25 & $3407-3579$ & $336-680$ & DEMUX \\
\hline 13269 & GULF SEAL & IPOD & IPOD-26 & $3579-3753$ & $680-28$ & DEMUX \\
\hline 13270 & GULF SEAL & IPOD & IPOD-27 & $3753-3925$ & $28-372$ & DEMUX \\
\hline 13271 & GULF SEAL & IPOD & IPOD-28 & $3925-3940$ & $372-402$ & DEMUX \\
\hline 13272 & GULF SEAL & IPOD & IPOD-29 & $3940-4111$ & $403-743$ & DEMUX \\
\hline 13273 & GULF SEAL & IPOD & IPOD-30 & $4111-4281$ & $743-83$ & DEMUX \\
\hline 13274 & GULF SEAL & IPOD & IPOD-31 & $4281-4414 \mathrm{~A}$ & $83-10$ & DEMUX \\
\hline 13275 & GULF SEAL & IPOD & IPOD-32 & $4414 A-4583$ & $10-348$ & DEMUX \\
\hline 13276 & GULF SEAL & IPOD & IPOD-33 & $4583-4779$ & $349-739$ & DEMUX \\
\hline 13277 & GULF SEAL & IPOD & IPOD-34 & $4779-4972$ & $739-125$ & DEMUX \\
\hline 13278 & GULF SEAL & IPOD & IPOD-35 & $4972-5058$ & $125-298$ & DEMUX \\
\hline 13279 & GULF SEAL & IPOD & IPOD-36 & $5059-5256$ & 299-694 & DEMUX \\
\hline 13280 & GULF SEAL & IPOD & IPOD-37 & $5256-5454$ & $694-90$ & DEMUX \\
\hline 13281 & GULF SEAL & IPOD & IPOD-38 & $5454-5652$ & $90-485$ & DEMUX \\
\hline 13282 & GULF SEAL & IPOD & IPOD-39 & $5652-5850$ & $485-881$ & DEMUX \\
\hline 13283 & GULF SEAL & IPOD & IPOD-40 & $5850-6047$ & $881-275$ & DEMUX \\
\hline 13284 & GULF SEAL & IPOD & IPOD-41 & $6047-6246$ & $275-673$ & DEMUX \\
\hline 13285 & GULF SEAL & IPOD & IPOD-42 & $6246-6422$ & $673-26$ & DEMUX \\
\hline
\end{tabular}




\begin{tabular}{|c|c|c|c|c|c|c|}
\hline SLOT & PROJECT & LINE & REEL & SPTS & FILES & TYPE \\
\hline 13286 & GULF SEAL & IPOD & IPOD-43 & $6422-6621$ & $27-424$ & DEMUX \\
\hline 13287 & GULF SEAL & IPOD & IPOD-44 & $6621-6748$ & $424-677$ & DEMUX \\
\hline 13288 & GULF SEAL & IPOD & IPOD-45 & $6748-6918$ & $678-18$ & DEMUX \\
\hline 13289 & GULF SEAL & IPOD & IPOD-46 & $6918-7100$ & $18-401$ & DEMUX \\
\hline 13290 & GULF SEAL & IPOD & IPOD-47 & $7100-7294$ & $401-790$ & DEMUX \\
\hline 13291 & GULF SEAL & IPOD & IPOD-48 & $7294-7483$ & $790-168$ & DEMUX \\
\hline 13292 & GULF SEAL & IPOD & IPOD-49 & $7483-7677$ & $168-555$ & DEMUX \\
\hline 13293 & GULF SEAL & IPOD & IPOD-50 & $7677-7681$ & $555-564$ & DEMUX \\
\hline 16597 & GULF SEAL & IPOD & IPOD-51 & $7682-7935$ & $565-71$ & DEMUX \\
\hline 16598 & GULF SEAL & IPOD & IPOD-52. & $7935-8188$ & $71-577$ & DEMUX \\
\hline 16599 & GULF SEAL & IPOD & IPOD-53 & $8188-8441$ & $577-83$ & DEMUX \\
\hline 16600 & GULF SEAL & IPOD & IPOD-54 & $8441-8695$ & $83-592$ & DEMUX \\
\hline 16601 & GULF SEAL & IPOD & IPOD-55 & $8695-8948$ & $592-98$ & DEMUX \\
\hline 16602 & GULF SEAL & IPOD & IPOD-56 & $8948-8967$ & $98-135$ & DEMUX \\
\hline 16603 & GULF SEAL & IPOD & IPOD-57 & $8967-9220$ & $135-641$ & DEMUX \\
\hline 16604 & GULF SEAL & IPOD & IPOD-58 & $9220-9295$ & $641-812$ & DEMUX \\
\hline 16605 & GULF SEAL & IPOD & IPOD-59 & $9296-9556$ & $813-334$ & DEMUX \\
\hline 16606 & GULF SEAL & IPOD & IPOD-60 & $9556-9810$ & $334-841$ & DEMUX \\
\hline 16607 & GULF SEAL & IPOD & IPOD-61 & $9810-10063$ & $841-347$ & DEMUX \\
\hline 16608 & GULF SEAL & IPOD & IPOD-62 & $10063-10316$ & $347-853$ & DEMUX \\
\hline 16609 & GULF SEAL & IPOD & IPOD-63 & $10316-10569$ & $853-360$ & DEMUX \\
\hline 16610 & GULF SEAL & IPOD & IPOD-64 & $10569-10823$ & $360-867$ & DEMUX \\
\hline 16611 & GULF SEAL & IPOD & IPOD-65 & $10823-10861$ & $867-943$ & DEMUX \\
\hline 16612 & GULF SEAL & IPOD & IPOD-66 & $10861-11108$ & $943-438$ & DEMUX \\
\hline 16613 & GULF SEAL & IPOD & IPOD-67 & $11108-11117$ & $438-456$ & DEMUX \\
\hline 16614 & GULF SEAL & IPOD & IPOD-68 & $11118-11328$ & $457-417$ & DEMUX \\
\hline 16615 & GULF SEAL & IPOD & IPOD-69 & $11328-11581$ & $417-923$ & DEMUX \\
\hline 16616 & GULF SEAL & IPOD & IPOD-70 & $11581-11827$ & $923-416$ & DEMUX \\
\hline 16617 & GULF SEAL & IPOD & IPOD-71 & $11827-12074$ & $416-910$ & DEMUX \\
\hline 16618 & GULF SEAL & IPOD & IPOD-72 & $12074-12320$ & $910-402$ & DEMUX \\
\hline 16619 & GULF SEAL & IPOD & IPOD-73 & $12320-12567$ & $402-895$ & DEMUX \\
\hline 16620 & GULF SEAL & IPOD & IPOD-74 & $12567-12820$ & $895-402$ & DEMUX \\
\hline 16621 & GULF SEAL & IPOD & IPOD-75 & $12820-12834$ & $402-430$ & DEMUX \\
\hline 16622 & GULF SEAL & IPOD & IPOD-76 & $12835-13057$ & $431-876$ & DEMUX \\
\hline 16623 & GULF SEAL & IPOD & IPOD-77 & $13057-13279$ & $876-319$ & DEMUX \\
\hline 16624 & GULF SEAL & IPOD & IPOD-78 & $13279-13501$ & $319-764$ & DEMUX \\
\hline 16625 & GULF SEAL & IPOD & IPOD-79 & $13501-13722$ & $764-206$ & DEMUX \\
\hline 16626 & GULF SEAL & IPOD & IPOD-80 & $13722-13944$ & $206-650$ & DEMUX \\
\hline 16627 & GULF SEAL & IPOD & IPOD-81 & $13944-14111$ & $650-984$ & DEMUX \\
\hline 16628 & GULF SEAL & IPOD & IPOD-82 & $14112-14363$ & $985-487$ & DEMUX \\
\hline 16629 & GULF SEAL & IPOD & IPOD-82B & $14242-14258$ & $246-277$ & DEMUX \\
\hline 16630 & GULF SEAL & IPOD & IPOD-83 & $14363-14616$ & $487-993$ & DEMUX \\
\hline 16631 & GULF SEAL & IPOD & IPOD-84 & $14616-14867$ & $993-496$ & DEMUX \\
\hline 16632 & GULF SEAL & IPOD & IPOD-85 & $14867-15120$ & $496-1$ & DEMUX \\
\hline 16633 & GULF SEAL & IPOD & IPOD-86 & $15120-15371$ & $1-504$ & DEMUX \\
\hline 16634 & GULF SEAL & IPOD & IPOD-87 & $15371-15624$ & $504-10$ & DEMUX \\
\hline 16635 & GULF SEAL & IPOD & IPOD-88 & $15624-15875$ & $10-512$ & DEMUX \\
\hline 16636 & GULF SEAL & IPOD & IPOD-89 & $15875-16128$ & $512-17$ & DEMUX \\
\hline 16637 & GULF SEAL & IPOD & IPOD-90 & $16128-16249$ & $17-259$ & DEMUX \\
\hline 16638 & GULF SEAL & IPOD & IPOD-91 & $16250-16454$ & $260-229$ & DEMUX \\
\hline 16639 & GULF SEAL & IPOD & IPOD-92 & $16454-16706$ & $229-733$ & DEMUX \\
\hline 16640 & GULF SEAL & IPOD & IPOD-93 & $16706-16959$ & $733-240$ & DEMUX \\
\hline 16641 & GULF SEAL & IPOD & IPOD-94 & $16959-17212$ & $240-746$ & DEMUX \\
\hline 16642 & GULF SEAL & IPOD & IPOD-95 & $17212-17465$ & $746-252$ & DEMUX \\
\hline 16643 & GULF SEAL & IPOD & IPOD-96 & $17465-17717$ & $252-755$ & DEMUX \\
\hline 16644 & GULF SEAL & IPOD & IPOD-97 & $17717-17970$ & $755-261$ & DEMUX \\
\hline 16645 & GULF SEAL & IPOD & IPOD-98 & $17970-18222$ & $261-765$ & DEMUX \\
\hline 16646 & GULF SEAL & IPOD & IPOD-99 & $18222-18475$ & $765-271$ & DEMUX \\
\hline 16647 & GULF SEAL & IPOD & IPOD-100 & $18475-18726$ & $271-773$ & DEMUX \\
\hline
\end{tabular}




\begin{tabular}{|c|c|c|c|c|c|c|}
\hline SLOT & PROJECT & LINE & REEL & SPTS & FILES & TYPE \\
\hline 16648 & GULF SEAL & IPOD & IPOD-101 & 18726-18979 & $773-279$ & DEMUX \\
\hline 16649 & GULF SEAL & IPOD & IPOD-102 & $18979-19197$ & $279-716$ & DEMUX \\
\hline 16650 & GULF SEAL & IPOD & IPOD-103 & $19198-19420$ & $717-161$ & DEMUX \\
\hline 16651 & GULF SEAL & IPOD & IPOD-104 & $19420-19643$ & $161-607$ & DEMUX \\
\hline 16652 & GULF SEAL & IPOD & IPOD-105 & $19643-19865$ & $607-51$ & DEMUX \\
\hline 16653 & GULF SEAL & IPOD & IPOD-106 & $19865-20087$ & $51-496$ & DEMUX \\
\hline 16654 & GULF SEAL & IPOD & IPOD-107 & 20087-20309 & $496-939$ & DEMUX \\
\hline 16655 & GULF SEAL & IPOD & IPOD-108 & $20309-20531$ & $939-383$ & DEMUX \\
\hline 16656 & GULF SEAL & IPOD & IPOD-109 & $20531-20753$ & $383-827$ & DEMUX \\
\hline 16657 & GULF SEAL & IPOD & IPOD-110 & $20753-20970$ & $827-262$ & DEMUX \\
\hline 16658 & GULF SEAL & IPOD & IPOD-111 & $20970-21180$ & $262-682$ & DEMUX \\
\hline 16659 & GULF SEAL & IPOD & IPOD-112 & $21181-21403$ & $683-128$ & DEMUX \\
\hline 16660 & GULF SEAL & IPOD & IPOD-113 & $21403-21626$ & $128-573$ & DEMUX \\
\hline 16661 & GULF SEAL & IPOD & IPOD-114 & $21626-21847$ & $573-16$ & DEMUX \\
\hline 16662 & GULF SEAL & IPOD & IPOD-115 & $21848-22053$ & $17-427$ & DEMUX \\
\hline 16663 & GULF SEAL & IPOD & IPOD-116 & $22053-22276$ & $427-874$ & DEMUX \\
\hline 16664 & GULF SEAL & IPOD & IPOD-117 & $22276-22498$ & $874-317$ & DEMUX \\
\hline 16665 & GULF SEAL & IPOD & IPOD-118 & $22498-22719$ & $317-759$ & DEMUX \\
\hline 16666 & GULF SEAL & IPOD & IPOD-119 & $22719-22941$ & $759-204$ & DEMUX \\
\hline 16667 & GULF SEAL & IPOD & IPOD-120 & $22941-23128$ & $204-257$ & DEMUX \\
\hline 16668 & GULF SEAL & IPOD & IPOD-121 & $23128-23346$ & $257-694$ & DEMUX \\
\hline 16669 & GULF SEAL & IPOD & IPOD-122 & $23346-23567$ & $694-135$ & DEMUX \\
\hline 16670 & GULF SEAL & IPOD & IPOD- 123 & $23567-23730$ & $135-462$ & DEMUX \\
\hline 16671 & GULF SEAL & IPOD & IPOD-124 & $23732-23954$ & $465-910$ & DEMUX \\
\hline 16672 & GULF SEAL & IPOD & IPOD- 125 & $23954-24173$ & $911-347$ & DEMUX \\
\hline 16673 & GULF SEAL & IPOD & IPOD-126 & $24173-24391$ & $348-784$ & DEMUX \\
\hline 16674 & GULF SEAL & IPOD & IPOD-127 & $24392-24614$ & $785-229$ & DEMUX \\
\hline 16675 & GULF SEAL & IPOD & IPOD-128 & $24614-24836$ & $229-673$ & DEMUX \\
\hline 16676 & GULF SEAL & IPOD & IPOD-129 & $24836-25057$ & $673-115$ & DEMUX \\
\hline 16677 & GULF SEAL & IPOD & IPOD-130 & $25057-25101$ & $115-204$ & DEMUX \\
\hline 16678 & GULF SEAL & IPOD & IPOD-131 & $25102-25325$ & $205-651$ & DEMUX \\
\hline 16679 & GULF SEAL & IPOD & IPOD-132 & $25325-25544$ & $651-90$ & DEMUX \\
\hline 16680 & GULF SEAL & IPOD & IPOD-133 & $25544-25767$ & $90-535$ & DEMUX \\
\hline 16885 & GULF SEAL & IPOD & IPOD-134 & $25767-25989$ & $535-979$ & DEMUX \\
\hline 16886 & GULF SEAL & IPOD & IPOD-135 & $25989-26210$ & $979-422$ & DEMUX \\
\hline 16887 & GULF SEAL & IPOD & IPOD-136 & $26210-26432$ & $422-866$ & DEMUX \\
\hline 16888 & GULF SEAL & IPOD & IPOD-137 & $26432-26647$ & $866-296$ & DEMUX \\
\hline 16889 & GULF SEAL & IPOD & IPOD-138 & $26647-26870$ & $296-741$ & DEMUX \\
\hline 16890 & GULF SEAL & IPOD & IPOD-139 & $26870-27091$ & $741-184$ & DEMUX \\
\hline 16891 & GULF SEAL & IPOD & IPOD-140 & 27091-27314 & $184-630$ & DEMUX \\
\hline 16892 & GULF SEAL & IPOD & IPOD-141 & $27314-27536$ & $630-74$ & DEMUX \\
\hline 16893 & GULF SEAL & IPOD & IPOD-142 & 27536-27759 & $74-520$ & DEMUX \\
\hline 16894 & GULF SEAL & IPOD & IPOD-143 & $27759-27766$ & $520-533$ & DEMUX \\
\hline 16895 & GULF SEAL & IPOD & IPOD-144 & $27766-27988$ & $533-977$ & DEMUX \\
\hline 16896 & GULF SEAL & IPOD & IPOD-145 & $27988-28211$ & $977-423$ & DEMUX \\
\hline 16897 & GULF SEAL & IPOD & IPOD-146 & $28211-28433$ & $423-867$ & DEMUX \\
\hline 16898 & GULF SEAL & IPOD & IPOD-147 & $28433-28655$ & $867-311$ & DEMUX \\
\hline 16899 & GULF SEAL & IPOD & IPOD-148 & $28655-28878$ & $311-757$ & DEMUX \\
\hline 16900 & GULF SEAL & IPOD & IPOD-149 & $28878-29100$ & $757-201$ & DEMUX \\
\hline 16901 & GULF SEAL & IPOD & IPOD-150 & $29100-29322$ & $201-646$ & DEMUX \\
\hline 16902 & GULF SEAL & IPOD & IPOD-151 & $29322-29544$ & $646-90$ & DEMUX \\
\hline 16903 & GULF SEAL & IPOD & IPOD- 152 & 29544-29768 & $90-537$ & DEMUX \\
\hline 16904 & GULF SEAL & IPOD & IPOD-153 & 29768-29886 & $537-774$ & DEMUX \\
\hline 16905 & GULF SEAL & IPOD & IPOD-154 & $29886-29911$ & $774-824$ & DEMUX \\
\hline 16906 & GULF SEAL & IPOD & IPOD-155 & $29912-30133$ & $825-269$ & DEMUX \\
\hline 16907 & GULF SEAL & IPOD & IPOD-156 & $30134-30356$ & $269-713$ & DEMUX \\
\hline 16908 & GULF SEAL & IPOD & IPOD-157 & $30356-30580$ & $713-162$ & DEMUX \\
\hline 16909 & GULF SEAL & IPOD & IPOD-158 & $30580-30803$ & $162-608$ & DEMUX \\
\hline & GULF SEAL & IPOD & IPOD-159 & $30803-31026$ & $608-53$ & DEMUX \\
\hline
\end{tabular}




$\begin{array}{llllccc}\text { SLOT } & \text { PROJECT } & \text { LINE } & \text { REEL } & \text { SPTS } & \text { FILES } & \text { TYPE } \\ & & & & & & \\ 16911 & \text { GULF SEAL } & \text { IPOD } & \text { IPOD-160 } & 31026-31244 & 53-490 & \text { DEMUX } \\ 16912 & \text { GULF SEAL } & \text { IPOD } & \text { IPOD-161 } & 31244-31467 & 490-936 & \text { DEMUX } \\ 16913 & \text { GULF SEAL } & \text { IPOD } & \text { IPOD-162 } & 31467-31688 & 936-378 & \text { DEMUX } \\ 16914 & \text { GULF SEAL } & \text { IPOD } & \text { IPOD-163 } & 31688-31912 & 378-825 & \text { DEMUX } \\ 16915 & \text { GULF SEAL } & \text { IPOD } & \text { IPOD-164 } & 31912-32133 & 825-268 & \text { DEMUX } \\ 16916 & \text { GULF SEAL } & \text { IPOD } & \text { IPOD-165 } & 32133-32355 & 268-711 & \text { DEMUX } \\ 16917 & \text { GULF SEAL } & \text { IPOD } & \text { IPOD-166 } & 32355-32576 & 711-153 & \text { DEMUX } \\ 16918 & \text { GULF SEAL } & \text { IPOD } & \text { IPOD-167 } & 32576-32798 & 153-598 & \text { DEMUX } \\ 16919 & \text { GULF SEAL } & \text { IPOD } & \text { IPOD-168 } & 32798-33019 & 598-40 & \text { DEMUX } \\ 16920 & \text { GULF SEAL } & \text { IPOD } & \text { IPOD-169 } & 33019-33241 & 40-484 & \text { DEMUX } \\ 16921 & \text { GULF SEAL } & \text { IPOD } & \text { IPOD-170 } & 33241-33463 & 484-927 & \text { DEMUX } \\ 16922 & \text { GULF SEAL } & \text { IPOD } & \text { IPOD-171 } & 33463-33686 & 927-374 & \text { DEMUX } \\ 16923 & \text { GULF SEAL } & \text { IPOD } & \text { IPOD-172 } & 33686-33909 & 374-819 & \text { DEMUX } \\ 16924 & \text { GULF SEAL } & \text { IPOD } & \text { IPOD-173 } & 33909-34110 & 819-222 & \text { DEMUX }\end{array}$

